OPEN ACCESS

Edited by:

Hiroshi Eguchi,

Kindai University, Japan

Reviewed by:

Hanaa ElZawawy,

Alexandria University, Egypt

Wei-Lin Wang,

Zhejiang University, China

*Correspondence:

Danian Chen

danianchen2006@qq.com orcid.org/0000-0002-6916-2978

${ }^{\dagger}$ These authors have contributed equally to this work

Specialty section:

This article was submitted to Microbiome in Health and Disease, a section of the journal

Frontiers in Cellular

and Infection Microbiology

Received: 11 July 2021 Accepted: 01 December 2021 Published: 22 December 2021

Citation:

Hou J, Tang Y, Chen $Y$ and Chen D (2021) The Role of the Microbiota in Graves' Disease and Graves' Orbitopathy. Front. Cell. Infect. Microbiol. 11:739707. doi: 10.3389/fcimb.2021.739707

\section{The Role of the Microbiota in Graves' Disease and Graves' Orbitopathy}

\author{
Jueyu Hou ${ }^{1,2 \dagger}$, Yunjing Tang ${ }^{1,2 \dagger}$, Yongjiang $\mathrm{Chen}^{3 \dagger}$ and Danian Chen ${ }^{1,2 *}$ \\ 1 The Research Laboratory of Ophthalmology and Vision Sciences, State Key Laboratory of Biotherapy, West China Hospital, \\ Sichuan University, Chengdu, China, ${ }^{2}$ The Department of Ophthalmology, West China Hospital, Sichuan University, \\ Chengdu, China, ${ }^{3}$ The School of Optometry and Vision Science, University of Waterloo, Waterloo, ON, Canada
}

Graves' disease (GD) is a clinical syndrome with an enlarged and overactive thyroid gland, an accelerated heart rate, Graves' orbitopathy (GO), and pretibial myxedema (PTM). GO is the most common extrathyroidal complication of GD. GD/GO has a significant negative impact on the quality of life. GD is the most common systemic autoimmune disorder, mediated by autoantibodies to the thyroid-stimulating hormone receptor (TSHR). It is generally accepted that GD/GO results from complex interactions between genetic and environmental factors that lead to the loss of immune tolerance to thyroid antigens. However, the exact mechanism is still elusive. Systematic investigations into GD/GO animal models and clinical patients have provided important new insight into these disorders during the past 4 years. These studies suggested that gut microbiota may play an essential role in the pathogenesis of GD/GO. Antibiotic vancomycin can reduce disease severity, but fecal material transfer (FMT) from GD/GO patients exaggerates the disease in GD/GO mouse models. There are significant differences in microbiota composition between GD/GO patients and healthy controls. Lactobacillus, Prevotella, and Veillonella often increase in GD patients. The commonly used therapeutic agents for GD/GO can also affect the gut microbiota. Antigenic mimicry and the imbalance of T helper 17 cells (Th17)/ regulatory $T$ cells (Tregs) are the primary mechanisms proposed for dysbiosis in GD/GO. Interventions including antibiotics, probiotics, and diet modification that modulate the gut microbiota have been actively investigated in preclinical models and, to some extent, in clinical settings, such as probiotics (Bifidobacterium longum) and selenium supplements. Future studies will reveal molecular pathways linking gut and thyroid functions and how they impact orbital autoimmunity. Microbiota-targeting therapeutics will likely be an essential strategy in managing GD/GO in the coming years.

Keywords: gut microbiota, Graves' disease, Graves' orbitopathy (GO), TSHR (thyroid-stimulating hormone receptor), Th17 and Treg cells, Lactobacillus, Prevotella, Veillonella

\section{INTRODUCTION}

Graves' disease (GD) is an autoimmune disorder characterized by the unique association of an enlarged and overactive thyroid gland, an accelerated heart rate, Graves' orbitopathy (GO), and Graves' dermopathy such as pretibial myxedema (PTM), in its typical presentation. GD is the most common cause of hyperthyroidism. The lifetime risk is about $3 \%$ for women and $0.5 \%$ for men 
(Davies et al., 2020). GD is mediated by autoantibodies to the thyroid-stimulating hormone receptor (TSHR). The TSHR is also expressed in orbital fibroblasts. GO is the most common extrathyroidal complication of GD (Covelli and Ludgate, 2017). About $25 \%-30 \%$ of GD patients have GO, but careful orbital imaging analysis can identify subtle orbital soft tissue abnormalities in $50 \%-70 \%$ of GD patients (Smith and Hegedüs, 2016; Perros et al., 2017; Taylor et al., 2020). The annual GO incidence is about 16 cases per 100,000 Europeans and 10 cases per 100,000 Japanese (Hiromatsu et al., 2014; Perros et al., 2017). GO is characterized by orbital tissue remodeling, retro-orbital inflammation, and glycosaminoglycan accumulation (Hansen et al., 1999). The major clinical features include periorbital edema, eyelid lag, proptosis, limited ocular movement, orbital disfigurement, and diplopia (Ludgate, 2020). Glucocorticoids are the primary treatment for GO at the active stage; smoking cessation, selenium supplements, and ocular lubricants are also helpful. Approximately $2 \%$ of GO patients will develop moderate to severe disease. These patients can have a visual loss due to corneal ulcers or GO-related optic neuropathy and eventually need decompression surgery (Davies et al., 2020). Thus, GO has a significant negative impact on the quality of life (Taylor et al., 2020).

GD is a multifactorial disease resulting from complex interactions between genetic and environmental factors that lead to the loss of immune tolerance to thyroid antigens. TSHR, T-cell-mediated immunity, and the mesenchymal stem cell properties of orbital fibroblasts have been implicated in the pathogenesis of GO (Taylor et al., 2020). However, the exact mechanism is still elusive. Some studies support the causative roles of microbiota in the pathogenesis of GD/GO (Kohling et al., 2017; Masetti and Ludgate, 2020).

Trillions of microorganisms exist on the mucosal and epidermal surfaces in the human body, such as skin, mouth, nose, sinuses, gut, respiratory tract, and ocular surface. These microbes are composed of bacteria, viruses, and fungi; usually do not harm; and are beneficial for the human body (Belkaid and Hand, 2014; Khan et al., 2019). The gastrointestinal tract is the primary interface in the human body to host microorganisms. Gut microbiota includes all microorganisms in the gastrointestinal mucosa and has about $10^{14}$ microbial cells (Szablewski, 2018). Gut microbiota can protect the host from pathogens, accelerate food digestion and mineral uptake (such as selenium, iron, and zinc), and modulate the immune system (Fröhlich and Wahl, 2019; Shivaji, 2019). Dysbiosis is an imbalance of the typical gut microbiota composition (Robles Alonso and Guarner, 2013). Dysbiosis can change the regulatory signaling of the immune system, resulting in pathological conditions of many organs (Gritz and Bhandari, 2015).

Indeed, increasing evidence has revealed that dysbiosis is closely connected to many diseases, including autoimmune diseases [e.g., rheumatoid arthritis (Gianchecchi and Fierabracci, 2019; Alghamdi and Redwan, 2021) and multiple sclerosis (Tsunoda, 2017; Zeng et al., 2019; Wang et al., 2021)], inflammatory diseases [e.g., ankylosing spondylitis (Ciccia et al., 2017; Wen et al., 2017; Berlinberg et al., 2021), infective endocarditis (Del Giudice et al., 2021), and inflammatory bowel disease (Kassam et al., 2018; Gianchecchi and Fierabracci, 2019; Pavel et al., 2021)], and ocular diseases [e.g., age-related macular degeneration (Rowan et al., 2017; Rinninella et al., 2018), diabetic retinopathy (Beli et al., 2018), dry eye (Cavuoto et al., 2019; Trujillo-Vargas et al., 2020), glaucoma (Chen et al., 2018; Doulberis et al., 2019), and uveitis (Fu et al., 2021)].

We will discuss the changes and potential mechanisms of the gut microbiota in the pathogenesis of GD/GO and comment on some possible therapeutic means to treat GD/GO by targeting the gut microbiome in this article.

\section{CHANGES OF THE GUT MICROBIOME IN GD/GO}

During the past 20 years, animal model studies have indicated a critical role of the gut microbiota in regulating innate and adaptive immune responses (Virili et al., 2021). Germ-free (GF) mouse models provide the most strong evidence to support that notion, including models of spontaneous ankylosing enteropathy (Rehakova et al., 2000), autoimmune arthritis (Wu et al., 2010), autoimmune encephalomyelitis (Lee et al., 2011), and autoimmune uveitis (Heissigerova et al., 2016; Fu et al., 2021). In these GF animal models, the disease incidence and severity are reduced under the GF environment, indicating the microbiota is crucial for the initiation and progression of these diseases (Vieira et al., 2014; Carding et al., 2015). This conclusion is further confirmed in clinical observations of many patients with ankylosing spondylitis (Ciccia et al., 2017; Wen et al., 2017), rheumatoid arthritis (Gianchecchi and Fierabracci, 2019), uveitis (Huang et al., 2018; Fu et al., 2021), and multiple sclerosis (Tsunoda, 2017; Zeng et al., 2019). GD is an autoimmune thyroid disease (AITD); the role of the gut microbiota in the pathogenesis of GD/GO was only discovered recently, based on both mouse models and clinical investigations (Table 1). These results are supported by findings that therapeutic agents of GD/GO (such as antithyroid drugs, glucocorticoids, immunosuppressants, and biologics) can also change the microbiota composition (Table 2).

\section{Animal Models of GD/GO}

Previously, a GD/GO animal model was established by transferring human TSHR-primed T cells into female BALB/c mice (Many et al., 1999). It was initially established in Brussels, Belgium. Many TSHRimmunized mice developed TSAb (thyroid-stimulating antibody) and GO-like phenotypes. However, this disease model could not be reproduced in Cardiff, UK (Baker et al., 2005). Because both animal facilities in Brussels and Cardiff are not pathogen-free, environmental microbial factors are possible reasons that TSHRinduced GO could not be generated in Cardiff. This result was the first scientific evidence suggesting that gut microbiota may be related to GD/GO pathogenesis (Baker et al., 2005; Masetti and Ludgate, 2020). The commonly used GD/GO mouse models are generally $\mathrm{BALB} / \mathrm{c}$ female mice, induced by electroporation of DNA 
TABLE 1 | Major references connecting the gut microbiome with GD/GO.

\begin{tabular}{|c|c|c|c|c|}
\hline Year & Study Type & Subjects & Major Findings & References \\
\hline 2018 & Experimental & BALB/c female, two locations & Disease-associated taxonomies explain the GD/GO variations observed & Masetti et al. (2018) \\
\hline 2018 & Experimental & BALB/c and C57BL/6J females & Big differences of BALB/c and C57BL/6J gut microbiota composition & $\begin{array}{l}\text { Moshkelgosha et al. } \\
\text { (2018) }\end{array}$ \\
\hline 2020 & Experimental & BALB/c female, FMT & FMT from GD donor increased the severity of GD & Su et al. (2020) \\
\hline 2021 & Experimental & $\begin{array}{l}\text { BALB/c female, microbiota } \\
\text { modification }\end{array}$ & $\begin{array}{l}\text { Vancomycin reduced, but FMT from GO donor increased the severity of GD/ } \\
\text { GO }\end{array}$ & $\begin{array}{l}\text { Moshkelgosha et al. } \\
\text { (2021) }\end{array}$ \\
\hline 2018 & Clinical-GD & $27 \mathrm{GD} / 12 \mathrm{HC}$ & Diversity reduced, F/B ratio increased & Ishaq et al. (2018) \\
\hline 2019 & Clinical-GD & $15 \mathrm{GD} / 15 \mathrm{HC}$ & Diversity reduced, F/B ratio increased & Yang M. et al. (2019) \\
\hline 2020 & Clinical-GD & $9 \mathrm{GD} / 11 \mathrm{HC}$ & Diversity reduced & $\begin{array}{l}\text { Cornejo-Pareja et al. } \\
\text { (2020) }\end{array}$ \\
\hline 2020 & Clinical-GD & $58 \mathrm{GD} / 63 \mathrm{HC}$ & Diversity reduced & Su et al. (2020) \\
\hline 2020 & Clinical-GD & $39 \mathrm{GD} / 17 \mathrm{HC}$ & Diversity reduced & Yan et al. (2020) \\
\hline 2021 & Clinical-GD & $15 \mathrm{GD} / 14 \mathrm{HC}$ & Diversity reduced & Chen et al. (2021) \\
\hline 2021 & Clinical-GD & $55 \mathrm{GD} / 48 \mathrm{HC}$ & Diversity unchanged, F/B ratio decreased & Chang et al. (2021) \\
\hline 2021 & Clinical-GD & $45 \mathrm{GD} / 59 \mathrm{HC}$ & Diversity reduced, F/B ratio decreased & Jiang et al. (2021) \\
\hline 2019 & Clinical-GO & $33 \mathrm{GO} / 32 \mathrm{HC}$ & Diversity reduced, F/B ratio decreased & Shi et al. (2019b) \\
\hline 2019 & Clinical-GO & $31 \mathrm{GO}$ & Links between the gut microbiota and GO-related traits are identified & Shi et al. (2019a) \\
\hline 2021 & $\begin{array}{l}\text { Clinical-GD/ } \\
\text { GO }\end{array}$ & $30 \mathrm{GD} / 33 \mathrm{GO} / 32 \mathrm{HC}$ & $\begin{array}{l}\text { Random forest algorithm can identify the three groups with } 70-80 \% \\
\text { accuracy }\end{array}$ & Shi et al. (2021) \\
\hline
\end{tabular}

GD, Graves' disease; GO, Graves' orbitopathy; F/B ratio, Firmicutes/Bacteroidetes ratio; HC, healthy controls.

plasmids expressing human TSHR A-subunit (Moshkelgosha et al., 2013) or injection of adenovirus expressing human TSHR Asubunit (Ad-TSHR289) (Chen et al., 2006). Both electroporation and Ad-TSHR289 can successfully induce GO-like phenotypes (Zhao et al., 2011).

\section{GD/GO Mouse Models in Two Locations (Essen and London)}

A recent study investigated the TSHR plasmid-immunized mice in two locations (Essen in Germany and London in the UK) by $16 \mathrm{~S}$ rRNA gene sequencing and routine microbiological tests (Berchner-Pfannschmidt et al., 2016; Masetti et al., 2018). These female BALB/c mice showed different gut microbiota compositions between these two SPF (specific pathogen-free) facilities. Essen mice had more abundant Lactobacillaceae, Ruminococcaceae, and
Porphyromonadaceae, but did not have Bifidobacteria. The Firmicutes : Bacteroidetes (F/B) ratio changed in TSHR mice of both locations. Orbital adipogenesis in Essen mice was correlated positively with Firmicutes OTUs (operational taxonomic units) and negatively with Bacteroidetes phyla. Disease-associated taxonomies have been identified and explained the clinical differences observed between Essen and London (Figure 1A). These findings suggest that gut microbiota may modulate the clinical heterogeneity of GD/ GO in TSHR-immunized mice (Masetti et al., 2018).

\section{GD/GO Models in Two Mouse Strains (C57BL/6 and BALB/c)}

After being immunized with TSHR plasmids, female C57BL/6 mice had produced both TSAb and TSBAb (TSH stimulating blocking antibody). However, none of these mice had GD or any

TABLE 2 | Major references connecting the gut microbiome with therapeutic agents for GD/GO.

\begin{tabular}{|c|c|c|c|c|}
\hline Year & Study Type & Therapeutic Agent & Subjects & References \\
\hline 2020 & Experimental & PTU & Adult male SD rat & Shin et al. (2020) \\
\hline 2020 & Experimental & PTU/MMl & Adult female SD rat & Sun et al. (2020) \\
\hline 2020 & Clinical & PTU/MMl & GD patient & Sun et al. (2020) \\
\hline 2021 & Clinical & $\mathrm{MMl}$ & GD patient & Chen et al. (2021) \\
\hline 2021 & Clinical & $\mathrm{MMl}$ & GD patient & Huo et al. (2021) \\
\hline 2011 & Experimental & Stress (increased steroid) & Mouse & Bailey et al. (2011) \\
\hline 2018 & Experimental & GCs & Bird & Noguera et al. (2018) \\
\hline 2019 & Clinical & GCs & Patient with GC-induced obesity & Qiu et al. (2019) \\
\hline 2020 & Experimental & GCs (short term) & Mouse & Zhao et al. (2020) \\
\hline 2020 & Experimental & GCs (long term) & Mouse & Schepper et al. (2020) \\
\hline 2021 & Clinical & $\mathrm{AZA}$ & Crohn's disease patient & Effenberger et al. (2021) \\
\hline 2018 & Experimental & MMF & Mouse & Flannigan et al. (2018) \\
\hline 2021 & Experimental & MMF & Spontaneously hypertensive rat (SHR) & Robles-Vera et al. (2021) \\
\hline 2019 & Clinical & Anti-TNF- $\alpha$ antibody & Crohn's disease patient & Yilmaz et al. (2019) \\
\hline 2021 & Clinical & Anti-TNF- $\alpha$ antibody & Enteropathic arthritis patient & Ditto et al. (2021) \\
\hline 2021 & Clinical & Anti-TNF- $\alpha$ antibody & Crohn's disease patient & Effenberger et al. (2021) \\
\hline
\end{tabular}

AZA, azathioprine; GD, Graves' disease; GCs, glucocorticoids; GO, Graves' orbitopathy; MMI, methimazole; MMF, mycophenolate mofetil; PTU, propylthiouracil; TNF- $\alpha$, antitumor necrosis factor $-\alpha$. 
A GD/GO mouse models in two locations (Essen and London)

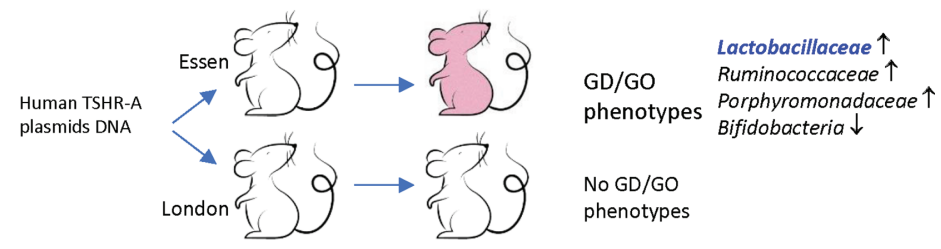

B GD/GO models in two mouse strains (C57BL/6 and BALB/C)

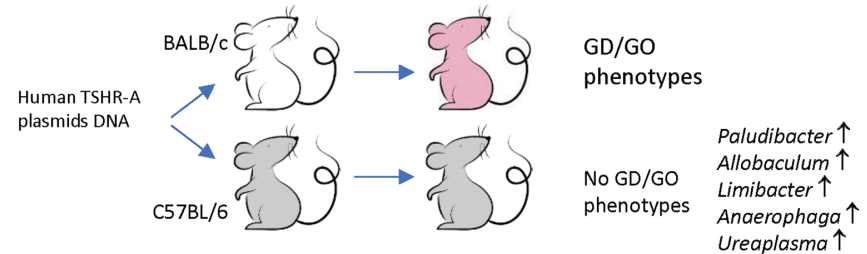

C Modulating the gut microbiota in GD/GO mouse models

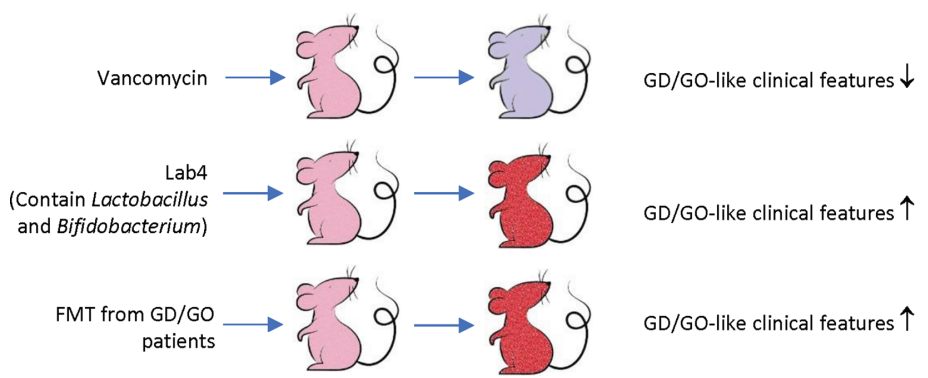

FIGURE 1 Animal model of Graves' disease (GD)/Graves' orbitopathy (GO) and gut microbiota. (A) BALB/c female mice were immunized with human TSHR-A plasmid DNA. The mice in Essen but not in London developed GD/GO phenotypes (pink). These mice have different gut microbiome profiles. Note, Essen mice have more Lactobacillaceae, which is also increased in GD patients and a component in Lab4. (B) BALB/c and C57BL/6 female mice were immunized with human TSHRA plasmid DNA. Only BALB/c but not C57BL/6 female mice developed GD/GO phenotypes (pink). These mice have different gut microbiome profiles. (C) Modify the gut microbiota of TSHR immunized mice (pink) by antibiotic vancomycin, probiotic Lab4, and fecal material transfer (FMT) from GD/GO patients, resulting in changed GD/GO-like clinical features (light purple or red). Derived from Berchner-Pfannschmidt et al. (2016); Masetti et al. (2018); Moshkelgosha et al. (2018); Su et al. (2020), and Moshkelgosha et al. (2021).

orbital soft tissue changes, while BALB/c female animals showed GD and GO-like phenotypes. Splenic T cells isolated from C57BL/6 mice did not grow upon TSHR stimulation and mainly produced IL-10, but not proinflammatory cytokines such as IFN- $\gamma$ (Moshkelgosha et al., 2018). 16S rRNA sequencing revealed increased beta-diversity between $\mathrm{BALB} / \mathrm{c}$ and $\mathrm{C} 57 \mathrm{BL} / 6 \mathrm{~J}$ gut microbiome and differential abundance of five genera (Paludibacter, Allobaculum, Limibacter, Anaerophaga, and Ureaplasma). These two mice strains had different correlations between gut microbiota and clinical manifestations; for instance, TSAb in C57BL/6J mice was correlated negatively with increased Limibacter. These results indicate that gut microbiota can modulate the immune activity, which explains different thyroid/orbit changes in different inbred mouse strains receiving TSHR immunization (Figure 1B).

\section{Modulating the Gut Microbiota in GD/GO Mouse Models}

To investigate whether the above-observed correlation indicates causation, the same research team altered the microbiota composition before the TSHR immunization by antibiotic vancomycin, probiotic Lab4, and fecal material transfer (FMT) from GO patients (Moshkelgosha et al., 2021). The antibiotic vancomycin was administered through drinking water, and probiotic Lab4 and FMT powder were administered through gavage. Vancomycin reduced the richness and diversity of gut microbiota. It depleted Firmicutes genera but increased Bacteroides, thus reducing the $\mathrm{F} / \mathrm{B}$ ratio. It also significantly reduced $\mathrm{CD} 4{ }^{+} \mathrm{CD} 25^{+}$regulatory $\mathrm{T}$ cells (Tregs) in orbital lymph nodes and GD/GO-like clinical features. TSHR mice receiving FMT from GO patients had a similar microbiota composition with their donors at the early stage after the transfer. GD-like features and orbital brown adipose tissue (BAT) volumes increased after FMT.

Lab4 are lactic acid bacteria that have been isolated from the gut flora of healthy humans. It combines four strains of lactic acid bacteria, including two strains of Lactobacillus acidophilus, one Bifidobacterium animalis subsp. lactis, and a Bifidobacterium bifidum. Despite Lab4 containing two Bifidobacteria species, none of them can be detected in any mice fed with Lab4, and 
the reason was unknown. Lab4 increased Tregs in orbital lymph nodes only in mice without TSHR immunization but not in mice receiving TSHR immunization. Lab4 exacerbated TSHR-induced autoimmune GD and GO-like phenotypes. Thus, although Lab4 increases orbital Tregs in normal mice, it cannot prevent TSHRinduced tolerance breakdown (Moshkelgosha et al., 2021).

In another BALB/c mouse model injected with Ad-TSHR289, FMT from GD patients before TSHR immunization also increased total T4, thyrotropin receptor antibody (TRAb), and IL-17A and doubled the GD incidence (Su et al., 2020). These findings suggest that the gut microbiota is involved in the development of GD/GO-like phenotypes (Figure 1C).

In summary (Table $\mathbf{1}$ and Figure 1), experimental GD/GO models of the same mouse strain but housed in two different animal facilities (Masetti et al., 2018), or different mouse strains from the same animal facility (Moshkelgosha et al., 2018), revealed significant differences in gut microbiota composition which explain variations in clinical manifestations. Modulating gut microbiota can change the incidence and severity of GD/GO
(Su et al., 2020; Moshkelgosha et al., 2021). These results uncovered a crucial role of gut microbiota in initiating and developing GD/GO mouse models (Masetti and Ludgate, 2020).

\section{Changes of the Gut Microbiome in GD/GO Patients}

There have been more than 10 clinical observational studies since 2018 comparing the gut microbiota of GD/GO patients to healthy controls (HCs). In total, fecal samples from 293 GD patients, 33 GO patients, and 271 healthy controls have been analyzed by $16 \mathrm{~S}$ rRNA gene sequencing (Table $\mathbf{1}$ ).

\section{Gut Microbiota in GD Patients}

Until July 2021, there are eight papers, including $263 \mathrm{GD} / 239 \mathrm{HC}$ samples, studying the gut microbiome of GD patients (Table $\mathbf{1}$ ). Overall, about 29 taxa are reported as differentially represented in GDs compared with HCs (Figure 2), and the gut microbial diversity decreased in most studies (Ishaq et al., 2018; Yang M. et al., 2019; Cornejo-Pareja et al., 2020; Su et al., 2020; Yan et al., 2020;

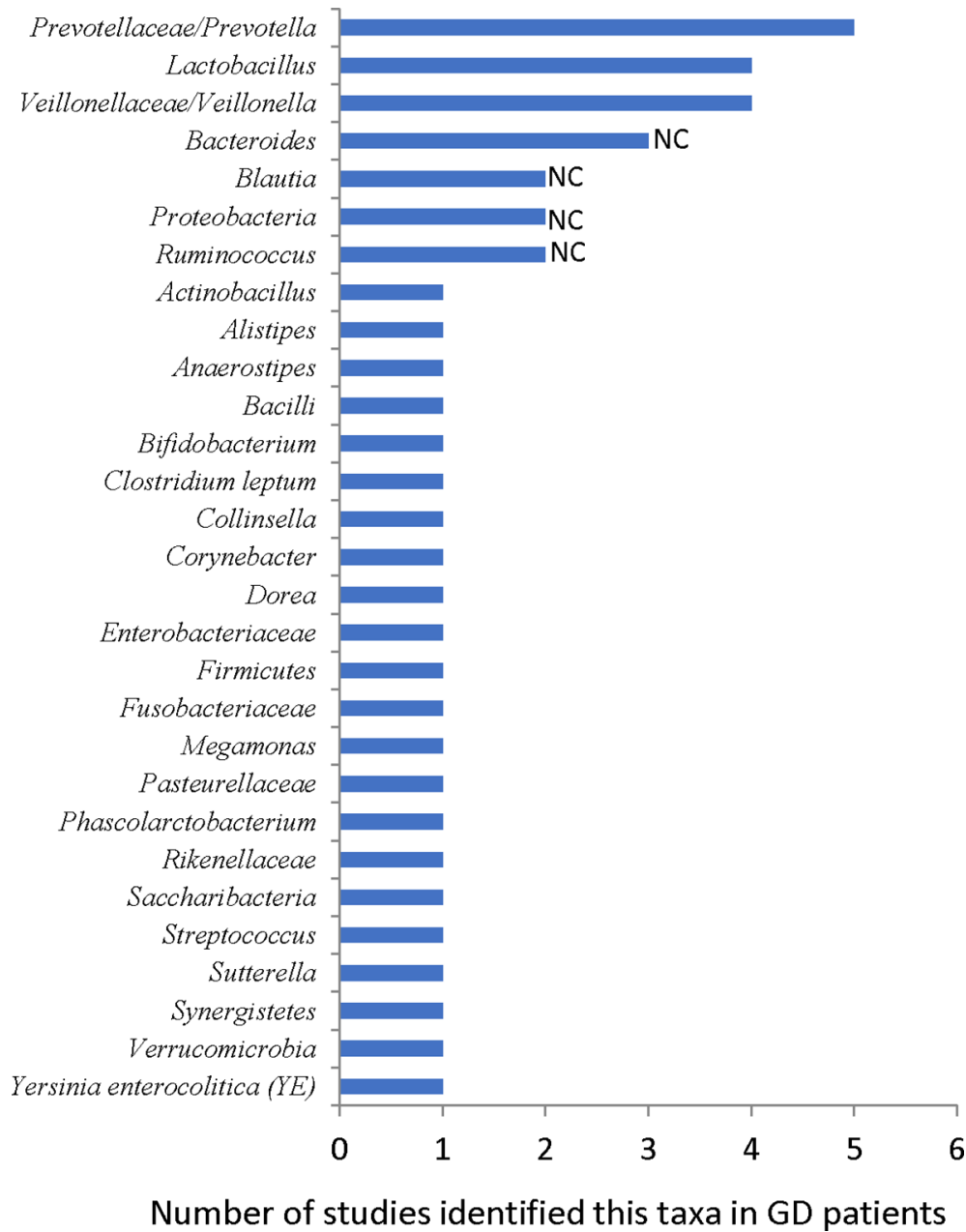

FIGURE 2 | Reported gut microbiota taxa changed in GD patients. NC: results are not consistent between studies. 
Changet al., 2021; Chen et al., 2021; Jiang et al., 2021). However, other results across these studies lack consistency. Differences in sample size, subject heterogeneity, study design, geographical location, and sequencing platform may contribute to the lack of reproducibility. Despite these limitations, several taxa were identified in three or more studies, including Prevotellaceae and Veillonellaceae at the family level and Bacteroides, Lactobacillus, Prevotella, and Veillonella at the genus level (Figure 2).

In a study comparing $27 \mathrm{GD}$ patients and $11 \mathrm{HCs}$, Prevotellaceae increased in GD patients (Ishaq et al., 2018). In another study with $58 \mathrm{GD}$ patients and $63 \mathrm{HCs}$, the random forest analysis showed that GD patients could be distinguished from HCs with $85 \%$ accuracy by three bacteria species, including Prevotella (Su et al., 2020). Prevotella also increased in GD patients in another study (Yan et al., 2020). Furthermore, Prevotellaceae and Prevotella were identified as the core microbiome of the GD group (Cornejo-Pareja et al., 2020). Moreover, they were found to be closely associated with GD patients (Chang et al., 2021). Prevotella has been linked with rheumatoid arthritis (RA), as specific antigens of Prevotella can shape or amplify immune responses in RA joints (Scher et al., 2013; Pianta et al., 2017; Pianta et al., 2021).

At the genus level, Bacteroides of GD patients decreased in two studies (Ishaq et al., 2018; Su et al., 2020) but increased in another one (Jiang et al., 2021). The Firmicutes and Bacteroidetes phyla are the major components of the human and mouse microbiota. The $\mathrm{F} / \mathrm{B}$ ratio of their abundance is often used as a dysbiosis marker. The F/B ratio increased in GD patients in two studies (Ishaq et al., 2018; Yang M. et al., 2019) but decreased in another two (Chang et al., 2021; Jiang et al., 2021), suggesting that this index may not be related to the pathogenesis of GD.

In three studies, Lactobacillus increased in GD patients (Yan et al., 2020; Chen et al., 2021; Jiang et al., 2021). Interestingly, the TRAb level was correlated positively with Lactobacillus, and the abundance of Lactobacillus decreased after oral methimazole (MMI) treatment (Chen et al., 2021). The TSHR plasmidimmunized GD/GO mice in Essen also had more abundant Lactobacillaceae (Figure 1) (Berchner-Pfannschmidt et al., 2016; Masetti et al., 2018). Lactobacillus is generally not pathogenic in the human body, and it is a recognized element in some probiotics such as Lab4 (Moshkelgosha et al., 2021). However, some studies also suggested that Lactobacillus can induce macrophages to secrete proinflammation cytokines, such as IL6 and TNF- $\alpha$ (Rocha-Ramírez et al., 2017), and the abundance of Lactobacillus increased in autoimmune hepatitis (Wei et al., 2020) and Crohn's disease (Wang et al., 2014; Effenberger et al., 2021). These results are consistent with the observed effects of Lab4 on mice. Lab4 increases orbital Tregs in normal mice but exacerbates TSHR-induced autoimmune GD and GOlike phenotypes (Figure 1) (Moshkelgosha et al., 2021).

The abundance of the family Veillonellaceae or the genus Veillonella increased in GD patients (Yan et al., 2020; Chang et al., 2021; Chen et al., 2021). Veillonella is a symbiotic bacterium in humans and can become a conditional pathogenic bacterium. For instance, Veillonella acts as a pathogen in various inflammatory diseases such as pneumonia (Shah et al., 2008). Veillonella is also associated with the disease activity of autoimmune hepatitis (Wei et al., 2020) and recurrent Crohn's disease (De Cruz et al., 2015). The role of Prevotella, Lactobacillus, and Veillonella in the pathogenesis of GD needs further investigation.

\section{Gut Microbiota in GO Patients}

There are fewer studies regarding the gut microbiota of GO patients. Until July 2021, there are only three reports from a single institute, including $30 \mathrm{GD}, 33 \mathrm{GO}$, and $30 \mathrm{HC}$ samples (Table 1). In one study, 33 active GO patients and $32 \mathrm{HCs}$ were compared (Shi et al., 2019b). Community diversity decreased in GO patients, consistent with most observations of GD patients (Ishaq et al., 2018; Yang M. et al., 2019; Cornejo-Pareja et al., 2020; Su et al., 2020; Chen et al., 2021; Jiang et al., 2021). The F/B ratio decreased in the GO group, consistent with some recent findings in GD patients (Su et al., 2020; Chang et al., 2021; Jiang et al., 2021). Metabolic-network-driven analysis of 31 hyperthyroid GO patients indicated that TRAb was associated with Prevotellaceae OTUs, CAS (clinical activity score) was associated with Bacteroides OTUs, and thyroglobulin autoantibodies were associated with OTUs from the Bacteroides stercoris species (Shi et al., 2019a). They also compared the original GO cohort (33 GO/32 HCs) with 30 GD patients taking antithyroid drugs. They identified bacterial phyla with different abundances between GD and GO patients, including Deinococcus-Thermus, Cyanobacteria, Chloroflexi, and Actinobacteria. At the genus level, Blautia, Anaerostipes, Dorea, and Butyricicoccus were more abundant in the GD group, while Subdoligranulum and Bilophila were more abundant in the GO group. Random forest analysis could differentiate GO patients, GD patients, and HCs with 70\%-80\% accuracy. DeinococcusThermus, Cyanobacteria, and Chloroflexi were the major taxa determining the classification accuracy (Shi et al., 2021). These results need to be confirmed with more studies on larger groups of patients.

In summary, clinical studies concluded that GD/GO patients and healthy controls have a much different gut microbiota composition. Whether these gut microbiota alterations in GD/ GO patients could contribute to disease pathogenesis or are just a consequence remains unknown. However, FMT from GD/GO patients significantly increased GD/GO incidence in the GD/GO mouse model, suggesting a fundamental pathogenic role of gut microbiota in the development of GD/GO (Su et al., 2020; Moshkelgosha et al., 2021).

\section{THE EFFECTS ON THE GUT MICROBIOTA OF THERAPEUTIC AGENTS FOR GD/GO}

The primary goal in the treatment of GD is restoring normal thyroid hormone levels. To reach this goal, antithyroid drugs (such as thionamides), radioiodine, and thyroidectomy are commonly used. The treatment of GO is stage-dependent. The anti-inflammatory agent is recommended for active progressive 
disease, and rehabilitative surgery is performed only in the stable inactive stage (Davies et al., 2020). Glucocorticoids (GCs) are the first-line treatment; if an insufficient response is observed after 6 weeks, second-line therapy, including immunosuppressants (such as mycophenolate mofetil and azathioprine) and biologic agents (such as rituximab, infliximab tocilizumab, and teprotumumab) should be considered (Davies et al., 2020; Taylor et al., 2020). Recent studies indicated that some of these therapeutic agents (including antithyroid drugs, glucocorticoids, immunosuppressants, and biologics) could also affect the gut microbiota (Table 2).

\section{Antithyroid Drugs and Gut Microbiota}

Methimazole (MMI) and propylthiouracil (PTU) are the commonly used antithyroid drugs (ATDs). ATD can change the gut microbiota structure in wild-type adult rats (Shin et al., 2020; Sun et al., 2020) and GD patients (Sun et al., 2020; Chen et al., 2021; Huo et al., 2021).

The effects of PTU on microbiota were studied in adult male SD rats (Shin et al., 2020). After treatment for 4 weeks, the alphadiversity did not change. However, Christensenellaceae, Tenericutes, and Mollicutes increased, while Ruminococcaceae, Prevotella, Mogibacteriaceae, Alcaligenaceae, Betaproteobacteria, Burkholderiales, Sutterella, and Ruminococcus decreased (Shin et al., 2020). The effects of MMI/PTU on microbiota were also measured in female adult SD rats (Sun et al., 2020). ATDs increased the alpha-diversity, different from the results in female rats (Shin et al., 2020). The ATD group had more Bacteroidetes, Proteobacteria, and Spirochaetae, but fewer Firmicutes at the phylum level and more Prevotellaceae and Ruminococcaceae but fewer Lactobacillaceae and Peptostreptococcaceae at the family level. Compared with the MMI group, the PTU group had more Spirochaetae at the phylum level and more Spirochaetaceae and Clostridiaceae_1 but less Lachnospiraceae and Rikenellaceae at the family level. The microbial dysbiosis index (MDI) increased after ATD treatment, indicating that the gut microbiota structure was disturbed in the treatment group. The MDI of the MMI group was higher than that of the PTU group (Sun et al., 2020).

ATDs can also affect the gut microbiota composition of GD patients. The fecal samples from 20 MMI-treated GD patients, 20 PTU-treated GD patients, and 50 healthy controls were analyzed by $16 \mathrm{~S}$ rRNA sequencing. The MMI group had a higher Ace index than the PTU group. The community diversity was different between the two drug treatment groups. The MMI group had more Firmicutes at the phylum level, while the PTU group had more Bacteroidetes. The MMI group had more Blautia and Escherichia-Shigella at the genus level, while the PTU group had more Bacteroides and Lachnoclostridium. The MDI and the $\mathrm{F} / \mathrm{B}$ ratio suggested that dysbiosis occurred in both drug-treated groups. Interestingly, ATD treatment reduced short-chain fatty acid (SCFA)-producing bacteria, including Faecalibacterium, Ruminococcaceae, Lactobacillus, and Blautia (Sun et al., 2020).

In a recent study with 15 GD patients, MMI treatment reduced the abundance of Blautia, Lactobacillus, and Streptococcus but increased Proteobacteria (Chen et al., 2021).
In another study with 8 GD patients, MMI treatment for 6 months also reduced the microbial Shannon index and Faecalibacterium prausnitzii, Ligilactobacillus salivarius, Lactococcus lactis, and some species of the genera Porphyromonas and Prevotella (Huo et al., 2021).

\section{Glucocorticoids and Gut Microbiota}

GCs are the first-line treatments for moderate to severe active GO. GCs are potent immune-modulating drugs with a number of side effects, such as GC-induced obesity or osteoporosis, but little is known about the effect of steroid treatment on gut microbiota in GO patients. In patients with GC-induced obesity, gut microbial diversity decreased, Firmicutes (e.g., genus Streptococcus) increased, and Bacteroidetes were depleted. Concomitantly, the SCFA level decreased in gut microbial metabolites of these patients (Qiu et al., 2019).

In animal models, GCs can change the composition of gut microbiota. In mice, dexamethasone increased the abundance of Actinobacteria, Bifidobacterium, and Lactobacillus compared with controls (Huang et al., 2015). A recent study found different results regarding Lactobacillus (Zhao et al., 2020). At the phyla level, short-term dexamethasone treatment increased the abundance of Firmicutes. At the genus level, dexamethasone increased the abundance of Lachnospiraceae, Oscillibacter, Ruminococcaceae, Ruminiclostridium, Anaerotruncus, and Butyricicoccus but reduced the abundance of Lactobacillus, Enterorhabdus, and Pseudomonas (Zhao et al., 2020).

It is also proven in mice that subcutaneous prednisolone implants for 8 weeks can promote dysbiosis, cause intestinal barrier leaks, and raise serum endotoxin levels. GCs reduced Verrucomicobiales and Bacteriodales and increased Clostridiales. These effects mediated the GC-induced osteoporosis (Schepper et al., 2020). In birds (yellow-legged gull Larus michahellis), corticosterone implants reduced Mycoplasma and Microvirga, which were potentially pathogenic avian bacteria, and increased Firmicutes, which was beneficial for birds (Noguera et al., 2018).

GCs can also alter the gut microbiota through changes in brain function. Stress can increase serum corticosteroid levels; stress also changes the mouse microbiome, and it reduces intestinal Bacteroides while increasing the relative abundance of bacteria in the genus Clostridium (Bailey et al., 2011). This effect is similar to the results of long-term subcutaneous prednisolone implants (Schepper et al., 2020).

\section{Immunosuppressant Drugs Have a Direct Effect on Microbiota}

Recent clinical trials concluded that combining steroids with immunosuppressant drugs (azathioprine and mycophenolate mofetil) has beneficial effects for GO patients (Kahaly et al., 2018; Rajendram et al., 2018). While these effects are generally attributed to their immunosuppressive activity, azathioprine (AZA) and mycophenolate mofetil (MMF) directly affect the microbiota. In liver cells, AZA is metabolized to 6mercaptopurine (MP), which can inhibit the maturation of $\mathrm{B}$ and T lymphocytes and the synthesis of DNA/RNA and proteins in immune cells. AZA can inhibit the growth of Campylobacter 
concisus, Bacteroides fragilis, Bacteroides vulgatus, Escherichia coli, and Mycobacterium Avium paratuberculosis (Shin and Collins, 2008; Antoniani et al., 2013; Liu et al., 2017). AZA is commonly used to treat inflammatory bowel disease (IBD). AZA can restore intestinal microbial diversity in patients with Crohn's disease by decreasing Proteobacteria but increasing Bacteroidetes (Effenberger et al., 2021).

MMF is an inhibitor of inosine- $5^{\prime}$-monophosphate dehydrogenase (IMPDH). MMF inhibits both T-cell and B-cell activities by blocking purine synthesis and is widely used in organ transplant recipients to reduce immune rejection (Ritter and Pirofski, 2009). MMF has some antibacterial (such as Staphylococcus epidermidis), antifungal (such as Cryptococcus, Aspergillus, Pneumocystis jirovekii, and Candida albicans), and antiviral (such as Camelpox virus, Cowpox virus, Monkeypox virus, and Vaccinia virus) activities, as it inhibits the synthesis of microbial DNA/RNA (Jones, 2020).

MMF treatment can cause gastrointestinal (GI) toxicity in organ transplant recipients. In mice, MMF reduced the overall gut microbial diversity and increased Proteobacteria. MMF-induced GI toxicity could be reversed or prevented using broad-spectrum antibiotics and was absent in germ-free animals (Flannigan et al., 2018; Taylor et al., 2019). Spontaneously hypertensive rats (SHR) are commonly used hypertension animal models. They have decreased gut microbial richness and acetate- and butyrateproducing bacteria and increased $\mathrm{F} / \mathrm{B}$ ratio and lactate-producing bacteria (Yang T. et al., 2019). MMF can reduce blood pressure in SHR (Rodríguez-Iturbe et al., 2002). MMF triggered substantial changes in the SHR microbiota taxa and reduced gut dysbiosis by reducing the $\mathrm{F} / \mathrm{B}$ ratio and lactate-producing bacteria and increasing acetate- and butyrate-producing bacteria (Robles-Vera et al., 2021).

\section{Biological Agents and Gut Microbiota}

Several biological agents that can be used as a novel therapy for GO patients. Even though there were very few studies regarding the effects of these agents on gut microbiota, their underlying pathways can interact with gut microbiota. For example, rituximab (RTX), a chimeric human-murine anti-CD20 monoclonal antibody, has been used to treat active moderatesevere GO for more than 15 years (Vannucchi et al., 2021). RTX eliminates orbital and peripheral B cells, therefore, reducing the production of antibodies. B-cell-produced $\operatorname{IgA}$ is the most abundant antibody in the mucosa; secretory $\operatorname{IgA}(\mathrm{SIgA})$ is secreted into the lumen of the gut. IgA can bind to multiple distinct taxonomic groups of the microbiota and is involved in the elimination, neutralization, and colonization of gut microbiota. IgA can also regulate bacterial gene expression (Weis and Round, 2021). Thus, RTX should have some effects on gut microbiota, but this has not been specifically addressed.

Tocilizumab, a humanized recombinant IL-6R monoclonal antibody, can improve disease activity and severity in corticosteroid-resistant GO patients (Perez-Moreiras et al., 2018; Sánchez-Bilbao et al., 2020). Infliximab, an antitumor necrosis factor (TNF)- $\alpha$ antibody, has been successfully used to treat sight-threatening GO (Durrani et al., 2005; Komorowski et al., 2007; Fallahi et al., 2021). Both IL-6 and TNF- $\alpha$ are monocyte-derived key proinflammation cytokines. Gut microbiota can modulate the production of these cytokines through their metabolites. The microbial tryptophan metabolite tryptophol potently influences cytokine production, which has strong inhibitory effects on the TNF- $\alpha$ response. On the other hand, palmitoleic acid can affect the production of monocyte-derived cytokines (TNF- $\alpha$, IL-1 $\beta$, IL-6) but not lymphocyte-derived cytokines (IFN- $\gamma$, IL-17, IL-22) (Schirmer et al., 2016). Lachnospiraceae and Ruminococcaceae families, typically producing SCFAs, define the frequently relapsing disease and poor treatment response to anti-TNF- $\alpha$ in Crohn's disease (Yilmaz et al., 2019). Like AZA, the anti-TNF- $\alpha$ antibody can restore intestinal microbial diversity in patients with Crohn's disease by decreasing Proteobacteria but increasing Bacteroidetes (Effenberger et al., 2021). In 20 enteropathic arthritis patients who followed a Mediterranean diet, anti-TNF- $\alpha$ antibody treatment for 6 months could increase Lachnospiraceae family and Coprococcus genus and also induce a decreasing trend in Proteobacteria and Gammaproteobacteria and an increasing trend in Clostridia (Ditto et al., 2021).

Teprotumumab, an insulin-like growth factor-1 receptor (IGFR-1) inhibiting monoclonal antibody, was approved in the USA to treat GO (Kahaly et al., 2021). Gut microbiota can modulate circulating IGF-1 in the host. Microbiota-derived metabolites such as SCFAs are sufficient to induce IGF-1 (Yan and Charles, 2018). How teprotumumab affects gut microbiota has not yet been explored.

In summary, the fact that ATDs, GCs, AZA, and MMF can change the microbiota composition is consistent with the notion that modifying the gut microbiota may reduce the severity of GD/GO, thus strengthening the concept that GD/GO and dysbiosis are tightly connected. The relationship between biological agents and gut microbiota needs further study in the future.

\section{HOW GUT MICROBIOTA AFFECT GD/GO DEVELOPMENT}

Two major mechanisms are proposed, namely, molecular/ antigenic mimicry and imbalance of proinflammation $\mathrm{T}$ helper 17 cells (Th17) and Treg cells (Figure 3).

\section{Antigenic Mimicry}

Yersinia enterocolitica (YE) and Helicobacter pylori (HP) were thought to be possible environmental risk factors for GD for a long time (Wolf et al., 1991). The structural or conformational similarity between different antigens can lead to cross-reactivity, also known as molecular or antigenic mimicry. Cross-reactivity between gut microbial peptides and self-antigens can produce autoreactive T cells and induce autoimmunity (Avni and Koren, 2018; Rojas et al., 2018; Wildner and Diedrichs-Möhring, 2020). If microbes have similar protein structures in their host, they will escape immune detection (Figure 3A). 
A

A Antigenic mimicry

Imbalance between Th17 and Tregs cells

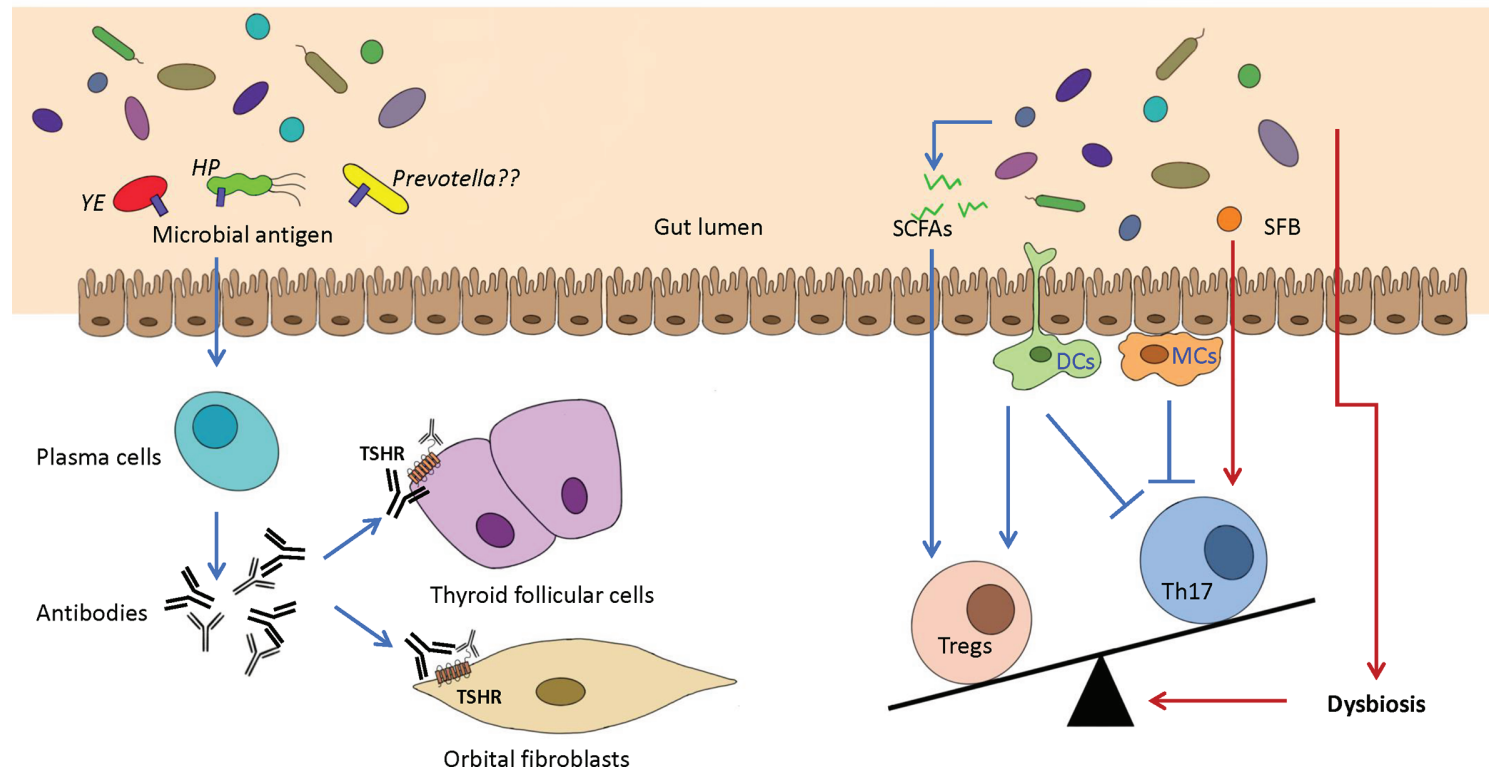

FIGURE 3 | Two potential proposed mechanisms of GD/GO are caused by dysbiosis of the gut microbiome. (A) Antigenic mimicry. Antigenic mimics in the gut microbiome, which have a highly similar structure or sequence with the autoantigens (e.g., TSHR), could activate plasma cells to produce antibodies that can bind TSHR on the thyroid follicular cells and orbital fibroblasts. Possible pathogenic microbes include Yersinia enterocolitica (YE), Helicobacter pylori (HP), and Prevotella. (B) Imbalance between Th17 and Tregs cells. Intestinal dysbiosis may cause the absence of beneficial microbiota and the reduction in beneficial anti-inflammatory metabolites such as SCFAs, which can increase the production of Tregs. SFB can promote the differentiation and maturation of Th17 cells. The imbalance between Th17 and Tregs cells can indirectly promote the progression of GD/GO. TSHR, thyroid-stimulating hormone receptor; SCFA, short-chain fatty acids; SFB,

segmented filamentous bacteria.

Such similarities can be shared by amino acid and nucleotide sequence or protein 3D structures (Miraglia and Colla, 2019). YE porin proteins have sequence similarity with TSHR and can stimulate B cells to produce autoantibodies to TSHR (Wang et al., 2010; Hargreaves et al., 2013). The IgG from YE-infected patients can induce GD-like changes in human thyroid structures, and GD patients have a higher YE infection rate than the healthy controls (Wolf et al., 1991). Although YE is considered involved in the development of GD, 16S rRNA gene sequencing did not detect it in GD patients. However, PCR analysis could easily detect YE in GD patients with diarrhea (Su et al., 2020).

$\mathrm{HP}$ in the human gastric mucosa can also affect the development of GD/GO (Bassi et al., 2010). Human TSHR is partly aligned with nine HP proteins such as NADH dehydrogenase subunit L, ABC transporter, and radical SAM protein (Figura et al., 2019). The most virulent $\mathrm{HP}$ strains express the cytotoxin-associated gene $\mathrm{A}$ antigens (CagA). GD patients have a much higher prevalence of $\mathrm{CagA}^{+}$HP than healthy controls (Bassi et al., 2010). While these clinical observations support that antigenic mimicry may trigger GD/GO, it has not been proven in any animal model yet.

Several recent studies revealed that Prevotellaceae and Prevotella consistently increase in GD patients (Ishaq et al., 2018; CornejoPareja et al., 2020; Su et al., 2020; Yan et al., 2020; Chang et al., 2021). Prevotella has been linked with rheumatoid arthritis (RA), as specific antigens of Prevotella, such as Pc-27 and $N$ acetylglucosamine-6-sulfatase (GNS), can trigger antigenic mimicry with RA joints (Scher et al., 2013; Pianta et al., 2017; Pianta et al., 2021). If these Prevotella-related antigens have similarities with human TSHR, they need further investigation.

\section{Imbalance Between Th17 and Treg Cells}

The gut-associated lymphoid tissue has many T-cell populations, including proinflammation helper $\mathrm{T}$ (Th) cells and antiinflammation Tregs. T helper cells include Th1, Th2, and Th17 cells. Th17 cells are the most important autoimmunity-related cells. Autoimmune diseases are closely related to abnormal Th17 cells (Bettelli et al., 2007; Pandiyan et al., 2019). Usually, Th17 and Tregs cells are in a dynamic balance to maintain the immune homeostasis of the gut mucosa. Gut microbiota can keep the balance between Th17/Tregs (Omenetti and Pizarro, 2015) (Figure 3B).

For instance, some gut microorganisms, such as Intestinimonas and Roseburia, can produce SCFAs, including primarily acetic acid, propionic acid, and butyric acid. SCFAs can increase Tregs in the gut mucosa (Smith et al., 2013). Segmented filamentous bacteria (SFB) promote the differentiation and maturation of Th17 cells (Ivanov et al., 2008; Hedblom et al., 2018). Gut microbiota can affect gut dendritic cells and macrophage cells. Gut dendritic cells can secrete transforming growth factor-beta (TGF- $\beta$ ), promoting the differentiation of 
Th17 and Treg cells. Dendritic cells also produce retinoic acid, which can promote Treg development but suppress Th17 cells. Gut macrophages can inhibit Th17 development by producing IL-2 and IL-10 (Masetti and Ludgate, 2020). Thus, dysbiosis may increase Th17 cells and suppress Treg production, which can cause many intestine inflammatory conditions and some extraintestinal diseases such as autoimmune uveitis (Zhuang et al., 2017).

As expected, GD patients have much less circulating $\mathrm{CD} 4^{+} \mathrm{Foxp}^{+}$Tregs but more CD $4^{+} \mathrm{IL}-17^{+}$Th17 cells (Qin et al., 2017; Su et al., 2020). Gas chromatography-mass spectrometry (GC-MS) analysis generated metabolic profiles of gut microbiota in GD patients and indicated that two important SCFAs (propionic acid and butyric acid) were significantly decreased in GD patients (Su et al., 2020). SCFA-producing Bacteroides fragilis YCH46 strain (B.f.S) was significantly reduced in GD patients. The culturing medium of B.f.S increased Tregs and IL-10 levels but reduced Th17 cells and IL-17A levels in peripheral blood mononuclear cells (PBMCs) from healthy individuals. B.f.S also exacerbated the imbalance of Treg/Th17 cells in GD patients (Su et al., 2020). GO patients also have much more Th17 cells and higher IL-17A expression than normal controls (Fang et al., 2016). These results are further confirmed by single-cell sequencing of retrobulbar tissues from GO patients (Fang et al., 2019). Single-cell sequencing identified six major cell clusters in retrobulbar tissues, including orbital fibroblasts and lymphocytes. In the $\mathrm{CD} 4^{+} \mathrm{CD} 8^{-} \mathrm{T}-$ cell subset, both IFN- $\gamma$-producing and IL-17A-producing T cells increased, but FoxP3 ${ }^{+}$Tregs decreased in GO orbits (Fang et al., 2019). Thus, increased Th17 and impaired Treg responses may be involved in GD/GO pathogenesis.

Removing $\mathrm{CD}^{+}{ }^{+} \mathrm{CD} 25^{+}$Tregs afforded some GD-resistant C57BL/6 mice susceptible to Ad-TSHR289 immunization and increased the GD severity in susceptible BALB/c mice. Removing $\mathrm{CD} 4^{+} \mathrm{CD} 25^{+}$Tregs also promoted TSAB production but suppressed thyroid-blocking antibody synthesis. These results indicate that Tregs are essential for Ad-TSHR289-induced GD phenotypes (Saitoh and Nagayama, 2006). However, the importance of Th17 cells in the immune response of the GD mouse model varies among different genetic backgrounds (Horie et al., 2011). BALB/c and NOD-H2(h4) mouse strains with wildtype (WT) or knockout (KO) IL-17 genes were immunized with Ad-TSHR289. BALB/c mice developed GD with WT or KO IL17 gene, but NOD-H2(h4) mice developed GD only in some animals with WT IL17 gene. This result suggested that IL-17 is essential for the GD development only in NOD-H2(h4) but not in BALB/c background (Horie et al., 2011).

These findings suggest that Th17/Treg imbalance is involved in developing GD/GO in some genetic backgrounds or ethnic groups (for instance, all these abovementioned clinical observations are from the Asian population).

\section{TARGETING THE GUT MICROBIOTA TO TREAT GD/GO}

Dysbiosis is closely related to the development of GD/GO; therapeutic approaches targeting the gut microbiota may provide potential benefits to GD/GO patients. Currently, antibiotics, probiotics, diet modifications, and fecal microbial transplantation are the four major strategies proposed.

\section{Antibiotics}

Antibiotics can change the gut microbiome. It was shown that oral antibiotic vancomycin could reduce the GD/GO severity in mouse models by reducing gut microbiota richness and diversity (Figure 1C). The reduced orbital pathology was correlated positively with Akkermansia (Moshkelgosha et al., 2021). As HP infection of the gastric mucosa is associated with GD through an increased inflammatory status and molecular mimicry, antiHP therapy may also benefit GD/GO patients (Figura et al., 2019).

\section{Probiotics}

Probiotics are live microorganisms with health benefits, which improve or restore the gut microbiota. Probiotics can promote the differentiation of Tregs, thus modifying the intestine immune homeostasis (Lin, 2019). Probiotics have been tested in GD/GO mouse models (Moshkelgosha et al., 2021) and GD patients (Huo et al., 2021).

The probiotic Lab4 is a consortium comprising of Lactobacillus and Bifidobacterium. Lab4 elevated the orbital CD $25^{+}$Treg cells but promoted the GD/GO phenotypes of TSHR-immunized mice (Moshkelgosha et al., 2021). One possible reason is that Lactobacillus in Lab4 may be pathogenic to GD as it frequently increases in fecal samples from GD patients (Yan et al., 2020; Chen et al., 2021; Jiang et al., 2021). These results also suggest that probiotics alone are not enough to suppress GD/GO development. Treatment with MMI and probiotic Bifidobacterium longum $\left(2 \times 10^{7} \mathrm{CFU}\right.$ per day) for 6 months improved thyroid function and significantly reduced the TRAb concentration of nine GD patients (Huo et al., 2021). This treatment increased Bifidobacterium adolescentis, Bifidobacterium angulatum, Bifidobacterium breve, B. longum, and Faecalibacterium prausnitzii and reduced Blautia hansenii, Clostridium estertheticum, and Klebsiella pneumoniae. Several microbial metabolic pathways were enriched in subjects receiving the probiotic B. longum treatment, including fatty acid biosynthesis, toluene degradation, phenylacetate degradation, and flavin biosynthesis. SCFAs also increased in these patients (Huo et al., 2021). The above results from animal models and GD patients support the idea that Lactobacillus may not benefit GD patients.

Future studies need to optimize the beneficial microbe stains in the probiotics formula; for instance, the formula should not include Lactobacillus, Prevotella, and Veillonella, as their abundance often increases in GD patients (Ishaq et al., 2018; Yang M. et al., 2019; Cornejo-Pareja et al., 2020; Su et al., 2020; Yan et al., 2020; Chang et al., 2021; Chen et al., 2021; Jiang et al., 2021).

\section{Diet Modifications and Selenium Supplements}

The diet can shape the microbiome composition. Culture and geographic-related diet differences can cause microbiome composition changes, for instance, Firmicutes enriched in the USA and Russia, Bacteroides spp. enriched in France and China, 
and Prevotella spp. enriched in Germany and India (Fröhlich and Wahl, 2019). Most GD patients have a reduced microbial diversity, which can be helped by a fiber-rich, low-calorie diet (Meijnikman et al., 2018). Diet with more vegetables increases SCFAs and Bifidobacteria, but animal fat increases the production of secondary bile acids (Ercolini and Fogliano, 2018). Diet with eicosapentaenoic acid (EPA) can inhibit IFN- $\gamma$ and IL-17 productions, thus attenuating experimental autoimmune encephalomyelitis (EAE) (Unoda et al., 2013).

The Mediterranean diet (MD) is consumed in countries bordering the Mediterranean sea and is characterized by a high intake of vegetables and fruits, legumes, and whole grains combined with a moderate amount of red wine and olive oil. It is well known that MD plays a protective role in preventing cardiovascular diseases, type 2 diabetes mellitus, obesity, Alzheimer's or Parkinson's disease, and cancer. MD was associated with a higher abundance of Bacteroidetes, Prevotellacea, and Prevotella and a lower concentration of Firmicutes and Lachnospiraceae. MD can also induce fecal propionate and butyrate (De Filippis et al., 2016; GutiérrezDíaz et al., 2016). As discussed above, Prevotellacea and Prevotella often increase in GD patients (Yan et al., 2020; Chang et al., 2021; Chen et al., 2021), and MD may not be a good choice for GD/GO patients. However, these results are mainly from research in Asia; if MD has any beneficial effects on GD/GO patients in the other geographic locations needs further study.

In the 2021 European Group on Graves' orbitopathy (EUGOGO) clinical practice guidelines for the medical management of GO, oral selenium supplementation is recommended for patients with mild GO (Bartalena et al., 2021). Mild GO patients from selenium-deficient areas can benefit from oral selenium supplementation. A double-blind, randomized clinical trial confirmed that sodium selenite could improve both the quality of life and overall ocular outcome and slow the progression in mild GO patients (Marcocci et al., 2011). These effects may be partially related to the role of selenium on gut microbiota. In adult C57BL/6 male mice, selenium in the diet can increase the microbiota diversity (Kasaikina et al., 2011), increase Turicibacter and Akkermansia, but reduce Mucispirillum (Qixiao Zhai et al., 2018). Turicibacter has been reported to display potential anti-inflammatory activities in the gut, and Akkermansia plays an essential role in the gut barrier protection, immune modulation, and metabolic regulation of the host (Qixiao Zhai et al., 2018). Akkermansia is also correlated positively with reduced orbital pathology in vancomycin-treated GO mouse models (Figure 1C) (Moshkelgosha et al., 2021). Thus, selenium supplementation increased the gut microbial diversity and positively modulated health beneficial microbes and negatively modulated the deleterious microbes in mice models. However, the effects of selenium supplementation on human gut microbiota are still unknown (Ferreira et al., 2021).

\section{Fecal Microbiota Transplantation}

Fecal microbiota transplantation (FMT) transfers fecal bacteria and other microbes from a healthy donor into the patient to replace their dysbiotic microbiota. FMT has been successfully used to treat Clostridium difficile infection in colitis by increasing the diversity of the host microbiota (Cheng et al., 2019). FMT has also been used to treat rheumatoid arthritis (Zeng et al., 2021). FMT from GD/GO patients can increase the severity of induced GD/GO-like features in TSHR immunization mouse models ( $\mathrm{Su}$ et al., 2020; Moshkelgosha et al., 2021), but if FMT from healthy donors can suppress established GD/GO manifestation has not been tested yet.

\section{CONCLUSIONS AND FUTURE DIRECTIONS}

The relationship between gut microbiota and GD/GO has been uncovered during the past 4 years. Oral antibiotic vancomycin reduces disease severity in GD/GO mouse models, but FMT from GD/GO patients exaggerates the disease. There are significant differences in microbiota composition between GD/ GO patients and healthy controls. Lactobacillus, Prevotella, and Veillonella often increase in GD patients. GCs are the first-line treatment for GO and can also change the composition of the microbiota. Two immunosuppression drugs (AZA and MMF) for GO have some antimicrobial properties; two AIDs (MMI and PTU) can change microbiota composition. Antigenic mimicry and imbalance of Th17/Tregs are likely the mechanisms for the effects of dysbiosis on GD/GO phenotypes.

Interventions including antibiotics, probiotics, and diet modification that modulate the gut microbiota have been actively investigated in preclinical models and clinical settings. However, only limited data exist on their effects on GD/GO patients. More research is needed to reveal molecular pathways linking gut and thyroid functions and how they impact orbital autoimmunity. For instance, the gut microbial features of GO patients need to be determined in more geographic locations; the effects of different probiotic formulas on GD/GO mouse model and patients need to be investigated; and how Lactobacillus, Prevotella, and Veillonella affect GD/GO phenotypes is still unknown. We believe microbiota-targeting therapeutics will be an important strategy in the management of GD/GO. This conclusion requires not only a thorough understanding of the distinct gut microbial composition and function of GD/GO patients but also carefully designed clinical trials.

\section{AUTHOR CONTRIBUTIONS}

JH, YT, YC, and DC conceived and designed the manuscript, and all authors wrote, edited, and approved the manuscript.

\section{FUNDING}

This study was supported by grants to DC from the National Natural Science Foundation of China (81870665, 82171063). 


\section{REFERENCES}

Alghamdi, M. A., and Redwan, E. M. (2021). Interplay of Microbiota and Citrullination in the Immunopathogenesis of Rheumatoid Arthritis. Probiotics Antimicrob. Proteins. doi: 10.1007/s12602-021-09802-7

Antoniani, D., Rossi, E., Rinaldo, S., Bocci, P., Lolicato, M., Paiardini, A., et al. (2013). The Immunosuppressive Drug Azathioprine Inhibits Biosynthesis of the Bacterial Signal Molecule Cyclic-Di-GMP by Interfering With Intracellular Nucleotide Pool Availability. Appl. Microbiol. Biotechnol. 97, 7325-7336. doi: $10.1007 / \mathrm{s} 00253-013-4875-0$

Avni, O., and Koren, O. (2018). Molecular (Me)micry? Cell Host Microbe 23, 576578. doi: 10.1016/j.chom.2018.04.012

Bailey, M. T., Dowd, S. E., Galley, J. D., Hufnagle, A. R., Allen, R. G., and Lyte, M. (2011). Exposure to a Social Stressor Alters the Structure of the Intestinal Microbiota: Implications for Stressor-Induced Immunomodulation. Brain Behav. Immun. 25, 397-407. doi: 10.1016/j.bbi.2010.10.023

Baker, G., Mazziotti, G., Von Ruhland, C., and Ludgate, M. (2005). Reevaluating Thyrotropin Receptor-Induced Mouse Models of Graves' Disease and Ophthalmopathy. Endocrinology 146, 835-844. doi: 10.1210/en.2004-1015

Bartalena, L., Kahaly, G. J., Baldeschi, L., Dayan, C. M., Eckstein, A., Marcocci, C., et al. (2021). The 2021 European Group on Graves' Orbitopathy (EUGOGO) Clinical Practice Guidelines for the Medical Management of Graves' Orbitopathy. Eur. J. Endocrinol. 185, G43-g67. doi: 10.1530/EJE-21-0479

Bassi, V., Santinelli, C., Iengo, A., and Romano, C. (2010). Identification of a Correlation Between Helicobacter Pylori Infection and Graves' Disease. Helicobacter 15, 558-562. doi: 10.1111/j.1523-5378.2010.00802.x

Beli, E., Yan, Y., Moldovan, L., Vieira, C. P., Gao, R., Duan, Y., et al. (2018). Restructuring of the Gut Microbiome by Intermittent Fasting Prevents Retinopathy and Prolongs Survival in Db/Db Mice. Diabetes 67, 1867-1879. doi: $10.2337 / \mathrm{db} 18-0158$

Belkaid, Y., and Hand, T. W. (2014). Role of the Microbiota in Immunity and Inflammation. Cell 157, 121-141. doi: 10.1016/j.cell.2014.03.011

Berchner-Pfannschmidt, U., Moshkelgosha, S., Diaz-Cano, S., Edelmann, B., Görtz, G. E., Horstmann, M., et al. (2016). Comparative Assessment of Female Mouse Model of Graves' Orbitopathy Under Different Environments, Accompanied by Proinflammatory Cytokine and T-Cell Responses to Thyrotropin Hormone Receptor Antigen. Endocrinology 157, 1673-1682. doi: 10.1210/en.2015-1829

Berlinberg, A. J., Regner, E. H., Stahly, A., Brar, A., Reisz, J. A., Gerich, M. E., et al. (2021). Multi 'Omics Analysis of Intestinal Tissue in Ankylosing Spondylitis Identifies Alterations in the Tryptophan Metabolism Pathway. Front. Immunol. 12, 587119. doi: 10.3389/fimmu.2021.587119

Bettelli, E., Oukka, M., and Kuchroo, V. K. (2007). T(H)-17 Cells in the Circle of Immunity and Autoimmunity. Nat. Immunol. 8, 345-350. doi: 10.1038/ ni0407-345

Carding, S., Verbeke, K., Vipond, D. T., Corfe, B. M., and Owen, L. J. (2015). Dysbiosis of the Gut Microbiota in Disease. Microb. Ecol. Health Dis. 26, 26191. doi: 10.3402/mehd.v26.26191

Cavuoto, K. M., Banerjee, S., and Galor, A. (2019). Relationship Between the Microbiome and Ocular Health. Ocul. Surf. 17, 384-392. doi: 10.1016/ j.jtos.2019.05.006

Chang, S. C., Lin, S. F., Chen, S. T., Chang, P. Y., Yeh, Y. M., Lo, F. S., et al. (2021). Alterations of Gut Microbiota in Patients With Graves' Disease. Front. Cell Infect. Microbiol. 11, 663131. doi: 10.3389/fcimb.2021.663131

Chen, C. R., Aliesky, H. A., Guo, J., Rapoport, B., and Mclachlan, S. M. (2006). Blockade of Costimulation Between T Cells and Antigen-Presenting Cells: An Approach to Suppress Murine Graves' Disease Induced Using Thyrotropin Receptor-Expressing Adenovirus. Thyroid 16, 427-434. doi: 10.1089/ thy.2006.16.427

Chen, H., Cho, K.-S., Vu, T. H. K., Shen, C.-H., Kaur, M., Chen, G., et al. (2018). Commensal Microflora-Induced T Cell Responses Mediate Progressive Neurodegeneration in Glaucoma. Nat. Commun. 9, 3209. doi: 10.1038/ s41467-018-05681-9

Cheng, Y. W., Phelps, E., Ganapini, V., Khan, N., Ouyang, F., Xu, H., et al. (2019). Fecal Microbiota Transplantation for the Treatment of Recurrent and Severe Clostridium Difficile Infection in Solid Organ Transplant Recipients: A Multicenter Experience. Am. J. Transplant. 19, 501-511. doi: $10.1111 /$ ajt.15058
Chen, J., Wang, W., Guo, Z., Huang, S., Lei, H., Zang, P., et al. (2021). Associations Between Gut Microbiota and Thyroidal Function Status in Chinese Patients With Graves' Disease. J. Endocrinol. Invest. 44 (9), 1913-1926. doi: 10.1007/ s40618-021-01507-6

Ciccia, F., Guggino, G., Rizzo, A., Alessandro, R., Luchetti, M. M., Milling, S., et al. (2017). Dysbiosis and Zonulin Upregulation Alter Gut Epithelial and Vascular Barriers in Patients With Ankylosing Spondylitis. Ann. Rheum. Dis. 76, 11231132. doi: 10.1136/annrheumdis-2016-210000

Cornejo-Pareja, I., Ruiz-Limón, P., Gómez-Pérez, A. M., Molina-Vega, M., Moreno-Indias, I., and Tinahones, F. J. (2020). Differential Microbial Pattern Description in Subjects With Autoimmune-Based Thyroid Diseases: A Pilot Study. J. Pers. Med. 10. doi: 10.3390/jpm10040192

Covelli, D., and Ludgate, M. (2017). The Thyroid, the Eyes and the Gut: A Possible Connection. J. Endocrinol. Invest. 40, 567-576. doi: 10.1007/s40618-016-0594-6

Davies, T. F., Andersen, S., Latif, R., Nagayama, Y., Barbesino, G., Brito, M., et al. (2020). Graves' Disease. Nat. Rev. Dis. Primers 6, 52. doi: 10.1038/s41572-0200184-y

De Cruz, P., Kang, S., Wagner, J., Buckley, M., Sim, W. H., Prideaux, L., et al. (2015). Association Between Specific Mucosa-Associated Microbiota in Crohn's Disease at the Time of Resection and Subsequent Disease Recurrence: A Pilot Study. J. Gastroenterol. Hepatol. 30, 268-278. doi: $10.1111 /$ jgh. 12694

De Filippis, F., Pellegrini, N., Vannini, L., Jeffery, I. B., La Storia, A., Laghi, L., et al. (2016). High-Level Adherence to a Mediterranean Diet Beneficially Impacts the Gut Microbiota and Associated Metabolome. Gut 65, 1812-1821. doi: 10.1136/gutjnl-2015-309957

Del Giudice, C., Vaia, E., Liccardo, D., Marzano, F., Valletta, A., Spagnuolo, G., et al. (2021). Infective Endocarditis: A Focus on Oral Microbiota Microorganisms 9. doi: 10.3390/microorganisms 9061218

Ditto, M. C., Parisi, S., Landolfi, G., Borrelli, R., Realmuto, C., Finucci, A., et al. (2021). Intestinal Microbiota Changes Induced by TNF-Inhibitors in IBDRelated Spondyloarthritis. RMD Open 7. doi: 10.1136/rmdopen-2021-001755

Doulberis, M., Polyzos, S. A., Papaefthymiou, A., Katsinelos, P., and Kountouras, J. (2019). Comments to the Editor Concerning the Paper Entitled "The Microbiome and Ophthalmic Disease" by Baim Et Al. Exp. Biol. Med. 244, 430-432. doi: 10.1177/1535370218824340

Durrani, O. M., Reuser, T. Q., and Murray, P. I. (2005). Infliximab: A Novel Treatment for Sight-Threatening Thyroid Associated Ophthalmopathy. Orbit 24, 117-119. doi: 10.1080/01676830590912562

Effenberger, M., Reider, S., Waschina, S., Bronowski, C., Enrich, B., Adolph, T. E., et al. (2021). Microbial Butyrate Synthesis Indicates Therapeutic Efficacy of Azathioprine in IBD Patients. J. Crohns Colitis 15, 88-98. doi: 10.1093/eccojcc/jjaa152

Ercolini, D., and Fogliano, V. (2018). Food Design To Feed the Human Gut Microbiota. J. Agric. Food Chem. 66, 3754-3758. doi: 10.1021/acs.jafc.8b00456

Fallahi, P., Ferrari, S. M., Elia, G., Ragusa, F., Paparo, S. R., Patrizio, A., et al. (2021). Cytokines as Targets of Novel Therapies for Graves' Ophthalmopathy. Front. Endocrinol. (Lausanne) 12, 654473. doi: 10.3389/fendo.2021.654473

Fang, S., Huang, Y., Wang, S., Zhang, Y., Luo, X., Liu, L., et al. (2016). IL-17a Exacerbates Fibrosis by Promoting the Proinflammatory and Profibrotic Function of Orbital Fibroblasts in TAO. J. Clin. Endocrinol. Metab. 101, 2955-2965. doi: 10.1210/jc.2016-1882

Fang, S., Huang, Y., Wang, N., Zhang, S., Zhong, S., Li, Y., et al. (2019). Insights Into Local Orbital Immunity: Evidence for the Involvement of the Th17 Cell Pathway in Thyroid-Associated Ophthalmopathy. J. Clin. Endocrinol. Metab. 104, 1697-1711. doi: 10.1210/jc.2018-01626

Ferreira, R. L. U., Sena-Evangelista, K. C. M., De Azevedo, E. P., Pinheiro, F. I., Cobucci, R. N., and Pedrosa, L. F. C. (2021). Selenium in Human Health and Gut Microflora: Bioavailability of Selenocompounds and Relationship With Diseases. Front. Nutr. 8, 685317. doi: 10.3389/fnut.2021.685317

Figura, N., Di Cairano, G., Moretti, E., Iacoponi, F., Santucci, A., Bernardini, G., et al. (2019). Helicobacter Pylori Infection and Autoimmune Thyroid Diseases: The Role of Virulent Strains. Antibiot. (Basel) 9. doi: 10.3390/ antibiotics 9010012

Flannigan, K. L., Taylor, M. R., Pereira, S. K., Rodriguez-Arguello, J., Moffat, A. W., Alston, L., et al. (2018). An Intact Microbiota is Required for the Gastrointestinal Toxicity of the Immunosuppressant Mycophenolate Mofetil. J. Heart Lung Transplant. 37, 1047-1059. doi: 10.1016/j.healun.2018.05.002 
Fröhlich, E., and Wahl, R. (2019). Microbiota and Thyroid Interaction in Health and Disease. Trends Endocrinol. Metab. 30, 479-490. doi: 10.1016/ j.tem.2019.05.008

$\mathrm{Fu}, \mathrm{X}$., Chen, Y., and Chen, D. (2021). The Role of Gut Microbiome in Autoimmune Uveitis. Ophthalmic Res. 64, 168-177. doi: 10.1159/000510212

Gianchecchi, E., and Fierabracci, A. (2019). Recent Advances on Microbiota Involvement in the Pathogenesis of Autoimmunity. Int. J. Mol. Sci. 20, 283. doi: $10.3390 / \mathrm{ijms} 20020283$

Gritz, E. C., and Bhandari, V. (2015). The Human Neonatal Gut Microbiome: A Brief Review. Front. Pediatr. 3, 17. doi: 10.3389/fped.2015.00017

Gutiérrez-Díaz, I., Fernández-Navarro, T., Sánchez, B., Margolles, A., and González, S. (2016). Mediterranean Diet and Faecal Microbiota: A Transversal Study. Food Funct. 7, 2347-2356. doi: 10.1039/C6FO00105J

Hansen, C., Rouhi, R., Förster, G., and Kahaly, G. J. (1999). Increased Sulfatation of Orbital Glycosaminoglycans in Graves' Ophthalmopathy. J. Clin. Endocrinol. Metab. 84, 1409-1413. doi: 10.1210/jc.84.4.1409

Hargreaves, C. E., Grasso, M., Hampe, C. S., Stenkova, A., Atkinson, S., Joshua, G. W., et al. (2013). Yersinia Enterocolitica Provides the Link Between ThyroidStimulating Antibodies and Their Germline Counterparts in Graves' Disease. J. Immunol. 190, 5373-5381. doi: 10.4049/jimmunol.1203412

Hedblom, G. A., Reiland, H. A., Sylte, M. J., Johnson, T. J., and Baumler, D. J. (2018). Segmented Filamentous Bacteria - Metabolism Meets Immunity. Front. Microbiol. 9, 1991. doi: 10.3389/fmicb.2018.01991

Heissigerova, J., Seidler Stangova, P., Klimova, A., Svozilkova, P., Hrncir, T., Stepankova, R., et al. (2016). The Microbiota Determines Susceptibility to Experimental Autoimmune Uveoretinitis. J. Immunol. Res., 2016, 5065703. doi: $10.1155 / 2016 / 5065703$

Hiromatsu, Y., Eguchi, H., Tani, J., Kasaoka, M., and Teshima, Y. (2014). Graves' Ophthalmopathy: Epidemiology and Natural History. Intern. Med. 53, 353360. doi: 10.2169/internalmedicine. 53.1518

Horie, I., Abiru, N., Saitoh, O., Ichikawa, T., Iwakura, Y., Eguchi, K., et al. (2011). Distinct Role of $\mathrm{T}$ Helper Type 17 Immune Response for Graves' Hyperthyroidism in Mice With Different Genetic Backgrounds. Autoimmunity 44, 159-165. doi: 10.3109/08916931003777247

Huang, E. Y., Inoue, T., Leone, V. A., Dalal, S., Touw, K., Wang, Y., et al. (2015). Using Corticosteroids to Reshape the Gut Microbiome: Implications for Inflammatory Bowel Diseases. Inflamm Bowel Dis. 21, 963-972. doi: 10.1097/MIB.0000000000000332

Huang, X., Ye, Z., Cao, Q., Su, G., Wang, Q., Deng, J., et al. (2018). Gut Microbiota Composition and Fecal Metabolic Phenotype in Patients With Acute Anterior Uveitis. Invest. Ophthalmol. Vis. Sci. 59, 1523-1531. doi: 10.1167/iovs.17-22677

Huo, D., Cen, C., Chang, H., Ou, Q., Jiang, S., Pan, Y., et al. (2021). Probiotic Bifidobacterium Longum Supplied With Methimazole Improved the Thyroid Function of Graves' Disease Patients Through the Gut-Thyroid Axis. Commun. Biol. 4, 1046. doi: 10.1038/s42003-021-02587-z

Ishaq, H. M., Mohammad, I. S., Shahzad, M., Ma, C., Raza, M. A., Wu, X., et al. (2018). Molecular Alteration Analysis of Human Gut Microbial Composition in Graves' Disease Patients. Int. J. Biol. Sci. 14, 1558-1570. doi: 10.7150/ ijbs. 24151

Ivanov, Ii, Frutos, R. D. E., Manel, N., Yoshinaga, K., Rifkin, D. B., Sartor, R. B., et al. (2008). Specific Microbiota Direct the Differentiation of IL-17-Producing T-Helper Cells in the Mucosa of the Small Intestine. Cell Host Microbe 4, 337349. doi: 10.1016/j.chom.2008.09.009

Jiang, W., Yu, X., Kosik, R. O., Song, Y., Qiao, T., Tong, J., et al. (2021). Gut Microbiota May Play a Significant Role in the Pathogenesis of Graves' Disease. Thyroid 31, 810-820. doi: 10.1089/thy.2020.0193

Jones, N. P. (2020). Immunosuppression in the Management of Presumed NonInfective Uveitis; Are We Sure What We are Treating? Notes on the Antimicrobial Properties of the Systemic Immunosuppressants. Ocul. Immunol. Inflamm. 28 (6), 994-1003. doi: 10.1080/09273948.2019.1643030

Kahaly, G. J., Douglas, R. S., Holt, R. J., Sile, S., and Smith, T. J. (2021). Teprotumumab for Patients With Active Thyroid Eye Disease: A Pooled Data Analysis, Subgroup Analyses, and Off-Treatment Follow-Up Results From Two Randomised, Double-Masked, Placebo-Controlled, Multicentre Trials. Lancet Diabetes Endocrinol. 9, 360-372. doi: 10.1016/S2213-8587(21)00056-5

Kahaly, G. J., Riedl, M., König, J., Pitz, S., Ponto, K., Diana, T., et al. (2018). Mycophenolate Plus Methylprednisolone Versus Methylprednisolone Alone in
Active, Moderate-to-Severe Graves' Orbitopathy (MINGO): A Randomised, Observer-Masked, Multicentre Trial. Lancet Diabetes Endocrinol. 6, 287-298. doi: 10.1016/S2213-8587(18)30020-2

Kasaikina, M. V., Kravtsova, M. A., Lee, B. C., Seravalli, J., Peterson, D. A., Walter, J., et al. (2011). Dietary Selenium Affects Host Selenoproteome Expression by Influencing the Gut Microbiota. FASEB J. 25, 2492-2499. doi: 10.1096/fj.11181990

Kassam, F., Gurry, T., Aldarmaki, A., Nguyen, T., Kassam, Z., Beck, P. L., et al. (2018). The Impact of the Gut Microbiome in Developing Uveitis Among Inflammatory Bowel Disease Patients: A Case-Control Study. Gastroenterology 154, S-415. doi: 10.1016/S0016-5085(18)31664-0

Khan, R., Petersen, F. C., and Shekhar, S. (2019). Commensal Bacteria: An Emerging Player in Defense Against Respiratory Pathogens. Front. Immunol. 10, 1203. doi: $10.3389 /$ fimmu.2019.01203

Kohling, H. L., Plummer, S. F., Marchesi, J. R., Davidge, K. S., and Ludgate, M. (2017). The Microbiota and Autoimmunity: Their Role in Thyroid Autoimmune Diseases. Clin. Immunol. 183, 63-74. doi: 10.1016/ j.clim.2017.07.001

Komorowski, J., Jankiewicz-Wika, J., Siejka, A., Lawnicka, H., Kłysik, A., Goś, R., et al. (2007). Monoclonal Anti-TNFalpha Antibody (Infliximab) in the Treatment of Patient With Thyroid Associated Ophthalmopathy. Klin. Oczna. 109, 457-460.

Lee, Y. K., Menezes, J. S., Umesaki, Y., and Mazmanian, S. K. (2011). Proinflammatory T-Cell Responses to Gut Microbiota Promote Experimental Autoimmune Encephalomyelitis. Proc. Natl. Acad. Sci. U. S. A. 108 (Suppl 1), 4615-4622. doi: 10.1073/pnas.1000082107

Lin, P. (2019). Importance of the Intestinal Microbiota in Ocular Inflammatory Diseases: A Review. Clin. Exp. Ophthalmol. 47, 418-422. doi: 10.1111/ ceo. 13493

Liu, F., Ma, R., Riordan, S. M., Grimm, M. C., Liu, L., Wang, Y., et al. (2017). Azathioprine, Mercaptopurine, and 5-Aminosalicylic Acid Affect the Growth of IBD-Associated Campylobacter Species and Other Enteric Microbes. Front. Microbiol. 8, 527. doi: 10.3389/fmicb.2017.00527

Ludgate, M. (2020). Fibrosis in Dysthyroid Eye Disease. Eye (Lond) 34, 279-284. doi: 10.1038/s41433-019-0731-5

Many, M. C., Costagliola, S., Detrait, M., Denef, F., Vassart, G., and Ludgate, M. C. (1999). Development of an Animal Model of Autoimmune Thyroid Eye Disease. J. Immunol. 162, 4966-4974.

Marcocci, C., Kahaly, G. J., Krassas, G. E., Bartalena, L., Prummel, M., Stahl, M., et al. (2011). Selenium and the Course of Mild Graves' Orbitopathy. N. Engl. J. Med. 364, 1920-1931. doi: 10.1056/NEJMoa1012985

Masetti, G., and Ludgate, M. (2020). Microbiome and Graves' Orbitopathy. Eur. Thyroid J. 9, 78-85. doi: 10.1159/000512255

Masetti, G., Moshkelgosha, S., Köhling, H. L., Covelli, D., Banga, J. P., BerchnerPfannschmidt, U., et al. (2018). Gut Microbiota in Experimental Murine Model of Graves' Orbitopathy Established in Different Environments may Modulate Clinical Presentation of Disease. Microbiome 6, 97. doi: 10.1186/s40168-0180478-4

Meijnikman, A. S., Gerdes, V. E., Nieuwdorp, M., and Herrema, H. (2018). Evaluating Causality of Gut Microbiota in Obesity and Diabetes in Humans. Endocr. Rev. 39, 133-153. doi: 10.1210/er.2017-00192

Miraglia, F., and Colla, E. (2019). Microbiome, Parkinson's Disease and Molecular Mimicry. Cells 8. doi: 10.3390/cells8030222

Moshkelgosha, S., Masetti, G., Berchner-Pfannschmidt, U., Verhasselt, H. L., Horstmann, M., Diaz-Cano, S., et al. (2018). Gut Microbiome in BALB/c and C57BL/6J Mice Undergoing Experimental Thyroid Autoimmunity Associate With Differences in Immunological Responses and Thyroid Function. Horm. Metab. Res. 50, 932-941. doi: 10.1055/a-0653-3766

Moshkelgosha, S., So, P. W., Deasy, N., Diaz-Cano, S., and Banga, J. P. (2013). Cutting Edge: Retrobulbar Inflammation, Adipogenesis, and Acute Orbital Congestion in a Preclinical Female Mouse Model of Graves' Orbitopathy Induced by Thyrotropin Receptor Plasmid-In Vivo Electroporation. Endocrinology 154, 3008-3015. doi: 10.1210/en.2013-1576

Moshkelgosha, S., Verhasselt, H. L., Masetti, G., Covelli, D., Biscarini, F., Horstmann, M., et al. (2021). Modulating Gut Microbiota in a Mouse Model of Graves' Orbitopathy and its Impact on Induced Disease. Microbiome 9, 45. doi: 10.1186/s40168-020-00952-4 
Noguera, J. C., Aira, M., Pérez-Losada, M., Domínguez, J., and Velando, A. (2018). Glucocorticoids Modulate Gastrointestinal Microbiome in a Wild Bird. R. Soc. Open Sci. 5, 171743. doi: 10.1098/rsos.171743

Omenetti, S., and Pizarro, T. T. (2015). The Treg/Th17 Axis: A Dynamic Balance Regulated by the Gut Microbiome. Front. Immunol. 6, 639. doi: 10.3389/ fimmu.2015.00639

Pandiyan, P., Bhaskaran, N., Zou, M., Schneider, E., Jayaraman, S., and Huehn, J. (2019). Microbiome Dependent Regulation of T(regs) and Th17 Cells in Mucosa. Front. Immunol. 10, 426. doi: 10.3389/fimmu.2019.00426

Pavel, F. M., Vesa, C. M., Gheorghe, G., Diaconu, C. C., Stoicescu, M., Munteanu, M. A., et al. (2021). Highlighting the Relevance of Gut Microbiota Manipulation in Inflammatory Bowel Disease. Diagn. (Basel) 11. doi: 10.3390/diagnostics11061090

Perez-Moreiras, J. V., Gomez-Reino, J. J., Maneiro, J. R., Perez-Pampin, E., Romo Lopez, A., Rodríguez Alvarez, F. M., et al. (2018). Efficacy of Tocilizumab in Patients With Moderate-To-Severe Corticosteroid-Resistant Graves Orbitopathy: A Randomized Clinical Trial. Am. J. Ophthalmol. 195, 181190. doi: 10.1016/j.ajo.2018.07.038

Perros, P., Hegedüs, L., Bartalena, L., Marcocci, C., Kahaly, G. J., Baldeschi, L., et al. (2017). Graves' Orbitopathy as a Rare Disease in Europe: A European Group on Graves' Orbitopathy (EUGOGO) Position Statement. Orphanet J. Rare Dis. 12, 72. doi: 10.1186/s13023-017-0625-1

Pianta, A., Arvikar, S., Strle, K., Drouin, E. E., Wang, Q., Costello, C. E., et al. (2017). Evidence of the Immune Relevance of Prevotella Copri, a Gut Microbe, in Patients With Rheumatoid Arthritis. Arthritis Rheumatol. 69, 964-975. doi: 10.1002/art.40003

Pianta, A., Chiumento, G., Ramsden, K., Wang, Q., Strle, K., Arvikar, S., et al. (2021). Identification of Novel, Immunogenic HLA-DR-Presented Prevotella Copri Peptides in Patients With Rheumatoid Arthritis: Patients With Rheumatoid Arthritis. Arthritis Rheumatol. 73 (12), 2200-2205. doi: 10. 1002/art.41807

Qin, J., Zhou, J., Fan, C., Zhao, N., Liu, Y., Wang, S., et al. (2017). Increased Circulating Th17 But Decreased CD4(+)Foxp3(+) Treg and CD19(+)CD1d(hi) CD5(+) Breg Subsets in New-Onset Graves' Disease. BioMed. Res. Int., 2017, 8431838. doi: 10.1155/2017/8431838

Qiu, D., Xia, Z., Deng, J., Jiao, X., Liu, L., and Li, J. (2019). Glucorticoid-Induced Obesity Individuals Have Distinct Signatures of the Gut Microbiome. Biofactors 45, 892-901. doi: 10.1002/biof.1565

Qixiao Zhai, S. C., Peng, L, Fengwei, T., Jianxin, Z., Hao, Z., and Wei, C. (2018). Effects of Dietary Selenium Supplementation on Intestinal Barrier and Immune Responses Associated With Its Modulation of Gut Microbiota. Environ. Sci. Technol. Lett. 5 (2), 724-730. doi: 10.1021/acs.estlett.8b00563

Rajendram, R., Taylor, P. N., Wilson, V. J., Harris, N., Morris, O. C., Tomlinson, M., et al. (2018). Combined Immunosuppression and Radiotherapy in Thyroid Eye Disease (CIRTED): A Multicentre, $2 \times 2$ Factorial, Double-Blind, Randomised Controlled Trial. Lancet Diabetes Endocrinol. 6, 299-309. doi: 10.1016/S2213-8587(18)30021-4

Rehakova, Z., Capkova, J., Stepankova, R., Sinkora, J., Louzecka, A., Ivanyi, P., et al. (2000). Germ-Free Mice do Not Develop Ankylosing Enthesopathy, a Spontaneous Joint Disease. Hum. Immunol. 61, 555-558. doi: 10.1016/S01988859(00)00122-1

Rinninella, E., Mele, M. C., Merendino, N., Cintoni, M., Anselmi, G., Caporossi, A., et al. (2018). The Role of Diet, Micronutrients and the Gut Microbiota in Age-Related Macular Degeneration: New Perspectives From the Gut(-)Retina Axis. Nutrients 10. doi: 10.3390/nu10111677

Ritter, M. L., and Pirofski, L. (2009). Mycophenolate Mofetil: Effects on Cellular Immune Subsets, Infectious Complications, and Antimicrobial Activity. Transpl. Infect. Dis. 11, 290-297. doi: 10.1111/j.1399-3062.2009.00407.x

Robles Alonso, V., and Guarner, F. (2013). Linking the Gut Microbiota to Human Health. Br. J. Nutr. 109 Suppl 2, S21-S26. doi: 10.1017/S0007114512005235

Robles-Vera, I., de la Visitación, N., Toral, M., Sánchez, M., Gómez-Guzmán, M., Jiménez, R., et al. (2021). Mycophenolate Mediated Remodeling of Gut Microbiota and Improvement of Gut-Brain Axis in Spontaneously Hypertensive Rats. BioMed. Pharmacother. 135, 111189. doi: 10.1016/ j.biopha.2020.111189

Rocha-Ramírez, L. M., Pérez-Solano, R. A., Castañón-Alonso, S. L., Moreno Guerrero, S. S., Ramírez Pacheco, A., García Garibay, M., et al. (2017). Probiotic Lactobacillus Strains Stimulate the Inflammatory Response and
Activate Human Macrophages. J. Immunol. Res., 2017, 4607491. doi: 10.1155/2017/4607491

Rodríguez-Iturbe, B., Quiroz, Y., Nava, M., Bonet, L., Chávez, M., Herrera-Acosta, J., et al. (2002). Reduction of Renal Immune Cell Infiltration Results in Blood Pressure Control in Genetically Hypertensive Rats. Am. J. Physiol. Renal Physiol. 282, F191-F201. doi: 10.1152/ajprenal.0197.2001

Rojas, M., Restrepo-Jimenez, P., Monsalve, D. M., Pacheco, Y., Acosta-Ampudia, Y., Ramirez-Santana, C., et al. (2018). Molecular Mimicry and Autoimmunity. J. Autoimmun. 95, 100-123. doi: 10.1016/j.jaut.2018.10.012

Rowan, S., Jiang, S., Korem, T., Szymanski, J., Chang, M. L., Szelog, J., et al. (2017). Involvement of a Gut-Retina Axis in Protection Against Dietary GlycemiaInduced Age-Related Macular Degeneration. Proc. Natl. Acad. Sci. U. S. A. 114, E4472-E4481. doi: 10.1073/pnas.1702302114

Saitoh, O., and Nagayama, Y. (2006). Regulation of Graves' Hyperthyroidism With Naturally Occurring CD4+CD25+ Regulatory T Cells in a Mouse Model. Endocrinology 147, 2417-2422. doi: 10.1210/en.2005-1024

Sánchez-Bilbao, L., Martínez-López, D., Revenga, M., López-Vázquez,, VallsPascual, E., Atienza-Mateo, B., et al. (2020). Anti-IL-6 Receptor Tocilizumab in Refractory Graves' Orbitopathy: National Multicenter Observational Study of 48 Patients. J. Clin. Med. 9 (9), 2816. doi: 10.3390/jcm9092816

Schepper, J. D., Collins, F., Rios-Arce, N. D., Kang, H. J., Schaefer, L., Gardinier, J. D., et al. (2020). Involvement of the Gut Microbiota and Barrier Function in Glucocorticoid-Induced Osteoporosis. J. Bone Miner Res. 35, 801-820. doi: $10.1002 / j b m r .3947$

Scher, J. U., Sczesnak, A., Longman, R. S., Segata, N., Ubeda, C., Bielski, C., et al. (2013). Expansion of Intestinal Prevotella Copri Correlates With Enhanced Susceptibility to Arthritis. Elife 2, e01202. doi: 10.7554/eLife.01202

Schirmer, M., Smeekens, S. P., Vlamakis, H., Jaeger, M., Oosting, M., Franzosa, E. A., et al. (2016). Linking the Human Gut Microbiome to Inflammatory Cytokine Production Capacity. Cell 167, 1125-1136.e1128. doi: 10.1016/ j.cell.2016.10.020

Shah, A., Panjabi, C., Nair, V., Chaudhry, R., and Thukral, S. S. (2008). Veillonella as a Cause of Chronic Anaerobic Pneumonitis. Int. J. Infect. Dis. 12, e115-e117. doi: $10.1016 /$ j.ijid.2008.03.018

Shi, T. T., Hua, L., Wang, H., and Xin, Z. (2019a). The Potential Link Between Gut Microbiota and Serum TRAb in Chinese Patients With Severe and Active Graves' Orbitopathy. Int. J. Endocrinol., 2019, 9736968. doi: 10.1155/2019/9736968

Shin, N. R., Bose, S., Wang, J. H., Nam, Y. D., Song, E. J., Lim, D. W., et al. (2020). Chemically or Surgically Induced Thyroid Dysfunction Altered Gut Microbiota in Rat Models. FASEB J. 34, 8686-8701. doi: 10.1096/ fj.201903091RR

Shin, S. J., and Collins, M. T. (2008). Thiopurine Drugs Azathioprine and 6Mercaptopurine Inhibit Mycobacterium Paratuberculosis Growth In Vitro. Antimicrob. Agents Chemother. 52, 418-426. doi: 10.1128/AAC.00678-07

Shivaji, S. (2019). Connect Between Gut Microbiome and Diseases of the Human Eye. J. Biosci. 44, 110. doi: 10.1007/s12038-019-9931-1

Shi, T. T., Xin, Z., Hua, L., Wang, H., Zhao, R. X., Yang, Y. L., et al. (2021). Comparative Assessment of Gut Microbial Composition and Function in Patients With Graves' Disease and Graves' Orbitopathy. J. Endocrinol. Invest. 44, 297-310. doi: 10.1007/s40618-020-01298-2

Shi, T. T., Xin, Z., Hua, L., Zhao, R. X., Yang, Y. L., Wang, H., et al. (2019b). Alterations in the Intestinal Microbiota of Patients With Severe and Active Graves' Orbitopathy: A Cross-Sectional Study. J. Endocrinol. Invest. 42, 967978. doi: 10.1007/s40618-019-1010-9

Smith, T. J., and Hegedüs, L. (2016). Graves' Disease. N Engl. J. Med. 375, 1552 1565. doi: 10.1056/NEJMra1510030

Smith, P. M., Howitt, M. R., Panikov, N., Michaud, M., Gallini, C. A., Bohlooly, Y. M., et al. (2013). The Microbial Metabolites, Short-Chain Fatty Acids, Regulate Colonic Treg Cell Homeostasis. Science 341, 569-573. doi: 10.1126/ science. 1241165

Sun, J., Zhao, F., Lin, B., Feng, J., Wu, X., Liu, Y., et al. (2020). Gut Microbiota Participates in Antithyroid Drug Induced Liver Injury Through the Lipopolysaccharide Related Signaling Pathway. Front. Pharmacol. 11, 598170. doi: 10.3389/fphar.2020.598170

Su, X., Yin, X., Liu, Y., Yan, X., Zhang, S., Wang, X., et al. (2020). Gut Dysbiosis Contributes to the Imbalance of Treg and Th17 Cells in Graves' Disease Patients by Propionic Acid. J. Clin. Endocrinol. Metab. 105 (11), 3526-3547. doi: $10.1210 /$ clinem/dgaa511 
Szablewski, L. (2018). Human Gut Microbiota in Health and Alzheimer's Disease. J. Alzheimers Dis. 62, 549-560. doi: 10.3233/JAD-170908

Taylor, M. R., Flannigan, K. L., Rahim, H., Mohamud, A., Lewis, I. A., Hirota, S. A., et al. (2019). Vancomycin Relieves Mycophenolate Mofetil-Induced Gastrointestinal Toxicity by Eliminating Gut Bacterial $\beta$-Glucuronidase Activity. Sci. Adv. 5, eaax2358. doi: 10.1126/sciadv.aax2358

Taylor, P. N., Zhang, L., Lee, R. W. J., Muller, I., Ezra, D. G., Dayan, C. M., et al. (2020). New Insights Into the Pathogenesis and Nonsurgical Management of Graves Orbitopathy. Nat. Rev. Endocrinol. 16, 104-116. doi: 10.1038/s41574019-0305-4

Trujillo-Vargas, C. M., Schaefer, L., Alam, J., Pflugfelder, S. C., Britton, R. A., and De Paiva, C. S. (2020). The Gut-Eye-Lacrimal Gland-Microbiome Axis in Sjogren Syndrome. Ocul. Surf. 18 (2), 335-344. doi: 10.1016/j.jtos.2019.10.006

Tsunoda, I. (2017). Lymphatic System and Gut Microbiota Affect Immunopathology of Neuroinflammatory Diseases, Including Multiple Sclerosis, Neuromyelitis Optica and Alzheimer's Disease. Clin. Exp. Neuroimmunol. 8, 177-179. doi: 10.1111/cen3.12405

Unoda, K., Doi, Y., Nakajima, H., Yamane, K., Hosokawa, T., Ishida, S., et al. (2013). Eicosapentaenoic Acid (EPA) Induces Peroxisome ProliferatorActivated Receptors and Ameliorates Experimental Autoimmune Encephalomyelitis. J. Neuroimmunol. 256, 7-12. doi: 10.1016/j.jneuroim. 2012.12.003

Vannucchi, G., Campi, I., Covelli, D., Currò, N., Lazzaroni, E., Palomba, A., et al. (2021). Efficacy Profile and Safety of Very Low-Dose Rituximab in Patients With Graves' Orbitopathy. Thyroid 31, 821-828. doi: 10.1089/thy.2020.0269

Vieira, S. M., Pagovich, O. E., and Kriegel, M. A. (2014). Diet, Microbiota and Autoimmune Diseases. Lupus 23, 518-526. doi: 10.1177/0961203313501401

Virili, C., Stramazzo, I., and Centanni, M. (2021). Gut Microbiome and Thyroid Autoimmunity. Best Pract. Res. Clin. Endocrinol. Metab. 35, 101506. doi: 10.1016/j.beem.2021.101506

Wang, W., Chen, L., Zhou, R., Wang, X., Song, L., Huang, S., et al. (2014). Increased Proportions of Bifidobacterium and the Lactobacillus Group and Loss of Butyrate-Producing Bacteria in Inflammatory Bowel Disease. J. Clin. Microbiol. 52, 398-406. doi: 10.1128/JCM.01500-13

Wang, X., Liang, Z., Wang, S., Ma, D., Zhu, M., and Feng, J. (2021). Role of Gut Microbiota in Multiple Sclerosis and Potential Therapeutic Implications. Curr. Neuropharmacol. doi: 10.2174/1570159X19666210629145351

Wang, Z., Zhang, Q., Lu, J., Jiang, F., Zhang, H., Gao, L., et al. (2010). Identification of Outer Membrane Porin F Protein of Yersinia Enterocolitica Recognized by Antithyrotopin Receptor Antibodies in Graves' Disease and Determination of its Epitope Using Mass Spectrometry and Bioinformatics Tools. J. Clin. Endocrinol. Metab. 95, 4012-4020. doi: 10.1210/jc.2009-2184

Wei, Y., Li, Y., Yan, L., Sun, C., Miao, Q., Wang, Q., et al. (2020). Alterations of Gut Microbiome in Autoimmune Hepatitis. Gut 69, 569-577. doi: 10.1136/ gutjnl-2018-317836

Weis, A. M., and Round, J. L. (2021). Microbiota-Antibody Interactions That Regulate Gut Homeostasis. Cell Host Microbe 29, 334-346. doi: 10.1016/ j.chom.2021.02.009

Wen, C., Zheng, Z., Shao, T., Liu, L., Xie, Z., Le Chatelier, E., et al. (2017). Quantitative Metagenomics Reveals Unique Gut Microbiome Biomarkers in Ankylosing Spondylitis. Genome Biol. 18, 142. doi: 10.1186/s13059-017-1271-6

Wildner, G., and Diedrichs-Möhring, M. (2020). Molecular Mimicry and Uveitis. Front. Immunol. 11, 580636. doi: 10.3389/fimmu.2020.580636

Wolf, M. W., Misaki, T., Bech, K., Tvede, M., Silva, J. E., and Ingbar, S. H. (1991). Immunoglobulins of Patients Recovering From Yersinia Enterocolitica
Infections Exhibit Graves' Disease-Like Activity in Human Thyroid Membranes. Thyroid 1, 315-320. doi: 10.1089/thy.1991.1.315

Wu, H. J., Ivanov, Ii., Darce, J., Hattori, K., Shima, T., Umesaki, Y., et al. (2010). GutResiding Segmented Filamentous Bacteria Drive Autoimmune Arthritis via T Helper 17 Cells. Immunity 32, 815-827. doi: 10.1016/j.immuni.2010.06.001

Yan, H. X., An, W. C., Chen, F., An, B., Pan, Y., Jin, J., et al. (2020). Intestinal Microbiota Changes in Graves' Disease: A Prospective Clinical Study. Biosci. Rep. 40. doi: 10.1042/BSR20191242

Yan, J., and Charles, J. F. (2018). Gut Microbiota and IGF-1. Calcif. Tissue Int. 102, 406-414. doi: 10.1007/s00223-018-0395-3

Yang, T., Aquino, V., Lobaton, G. O., Li, H., Colon-Perez, L., Goel, R., et al. (2019). Sustained Captopril-Induced Reduction in Blood Pressure Is Associated With Alterations in Gut-Brain Axis in the Spontaneously Hypertensive Rat. J. Am. Heart Assoc. 8, e010721. doi: 10.1161/JAHA.118.010721

Yang, M., Sun, B., Li, J., Yang, B., Xu, J., Zhou, X., et al. (2019). Alteration of the Intestinal Flora may Participate in the Development of Graves' Disease: A Study Conducted Among the Han Population in Southwest China. Endocr. Connect 8, 822-828. doi: 10.1530/EC-19-0001

Yilmaz, B., Juillerat, P., yås, O., Ramon, C., Bravo, F. D., Franc, Y., et al. (2019). Microbial Network Disturbances in Relapsing Refractory Crohn's Disease. Nat. Med. 25, 323-336. doi: 10.1038/s41591-018-0308-z

Zeng, Q., Junli, G., Liu, X., Chen, C., Sun, X., Li, H., et al. (2019). Gut Dysbiosis and Lack of Short Chain Fatty Acids in a Chinese Cohort of Patients With Multiple Sclerosis. Neurochem. Int. 129, 104468. doi: 10.1016/j.neuint.2019.104468

Zeng, J., Peng, L., Zheng, W., Huang, F., Zhang, N., Wu, D., et al. (2021). Fecal Microbiota Transplantation for Rheumatoid Arthritis: A Case Report. Clin. Case Rep. 9, 906-909. doi: 10.1002/ccr3.3677

Zhao, H., Jiang, X., and Chu, W. (2020). Shifts in the Gut Microbiota of Mice in Response to Dexamethasone Administration. Int. Microbiol. 23, 565-573. doi: 10.1007/s10123-020-00129-x

Zhao, S. X., Tsui, S., Cheung, A., Douglas, R. S., Smith, T. J., and Banga, J. P. (2011). Orbital Fibrosis in a Mouse Model of Graves' Disease Induced by Genetic Immunization of Thyrotropin Receptor cDNA. J. Endocrinol. 210, 369-377. doi: 10.1530/JOE-11-0162

Zhuang, Z., Wang, Y., Zhu, G., Gu, Y., Mao, L., Hong, M., et al. (2017). Imbalance of Th17/Treg Cells in Pathogenesis of Patients With Human Leukocyte Antigen B27 Associated Acute Anterior Uveitis. Sci. Rep. 7, 40414. doi: $10.1038 /$ srep 40414

Conflict of Interest: The authors declare that the research was conducted in the absence of any commercial or financial relationships that could be construed as a potential conflict of interest.

Publisher's Note: All claims expressed in this article are solely those of the authors and do not necessarily represent those of their affiliated organizations, or those of the publisher, the editors and the reviewers. Any product that may be evaluated in this article, or claim that may be made by its manufacturer, is not guaranteed or endorsed by the publisher.

Copyright (C) 2021 Hou, Tang, Chen and Chen. This is an open-access article distributed under the terms of the Creative Commons Attribution License (CC BY). The use, distribution or reproduction in other forums is permitted, provided the original author(s) and the copyright owner(s) are credited and that the original publication in this journal is cited, in accordance with accepted academic practice. No use, distribution or reproduction is permitted which does not comply with these terms. 